\title{
ON THE CAUCHY PROBLEM FOR A CLASS OF HYPERBOLIC OPERATORS WHOSE COEFFICIENTS DEPEND ONLY ON THE TIME VARIABLE
}

By

\author{
Seiichiro WAKABAYASHI
}

\begin{abstract}
In this paper we investigate the Cauchy problem for hyperbolic operators with double characteristics and hyperbolic operators of third order whose coefficients depend only on the time variable. And we give sufficient conditions for $C^{\infty}$ well-posedness.
\end{abstract}

\section{Introduction}

We say that a (partial differential) operator is an operator with timedependent coefficients if the coefficients of the operator depend only on the time variable. In [16] we studied the Cauchy problem for hyperbolic operators of second order with time-dependent coefficients. And we gave sufficient conditions for the Cauchy problem to be $C^{\infty}$ well-posed, assuming that the coefficients of the principal parts are real analytic functions of the time variable. These conditions are also necessary conditions if the space dimension is less than 3 , or if the coefficients of the principal parts of the operators are semi-algebraic functions (e.g., polynomials) of the time variable (see, also, [17]).

In this paper we shall deal with hyperbolic operators with time-dependent coefficients and double characteristics and give sufficient conditions for the Cauchy problem to be $C^{\infty}$ well-posed, imposing some conditions on the subprincipal symbols. Our conditions are generalizations of the conditions given in [16]. If one considers the Cauchy problem for hyperbolic operators of $\mathrm{m}$-th

2010 Mathematics Subject Classification: Primary 35L30; Secondary 35L25.

Key words and phrases: Cauchy problem, hyperbolic, $C^{\infty}$ well-posed, double characteristics, third order.

This research was partially supported by Grant-in-Aid for Scientific Research (No. 23540185), Japan Society for the Promotion of Science.

Received November 20, 2014.

Revised January 26, 2015. 
order, then one must impose some conditions not only on the subprincipal symbols but on the lower order symbols of order $k(1 \leq k \leq m-2)$, in general. So one needs to define the symbols of order $k(1 \leq k \leq m-2)$ corresponding to the subprincipal symbols in order to describe the conditions for $C^{\infty}$ wellposedness. To clarify the situation we consider hyperbolic operators of third order with time-dependent coefficients in this paper. In doing so, we shall define symbols of first order for operators of third order with time-dependent coefficients, which are called the sub-sub-principal symbols. We should note that Jackson [8] showed that the sub-sub-principal symbol can not be defined invariantly under canonical transformations. We shall prove $C^{\infty}$ well-posedness of the Cauchy problem for hyperbolic operators of third order with timedependent coefficients, imposing some conditions on the subprincipal symbols and the sub-sub-principal symbols.

Let $m \in \mathbf{N}$ and $P(t, \tau, \xi) \equiv \tau^{m}+\sum_{j=1}^{m} \sum_{|\alpha| \leq j} a_{j, \alpha}(t) \tau^{m-j} \xi^{\alpha}$ be a polynomial of $\tau$ and $\xi=\left(\xi_{1}, \ldots, \xi_{n}\right)$ of degree $m$ whose coefficients $a_{j, \alpha}(t)$ are $C^{\infty}$ functions of $t \in[0, \infty)$. Here $\alpha=\left(\alpha_{1}, \ldots, \alpha_{n}\right) \in\left(\mathbf{Z}_{+}\right)^{n}$ is a multi-index, $|\alpha|=\sum_{j=1}^{n} \alpha_{j}$ and $\xi^{\alpha}=\xi_{1}^{\alpha_{1}} \cdots \xi_{n}^{\alpha_{n}}$, where $\mathbf{Z}_{+}=\mathbf{N} \cup\{0\} \quad(=\{0,1,2,3, \ldots\})$. We consider the Cauchy problem

$$
\left\{\begin{array}{l}
P\left(t, D_{t}, D_{x}\right) u(t, x)=f(t, x) \quad \text { in }[0, \infty) \times \mathbf{R}^{n} \\
\left.D_{t}^{j} u(t, x)\right|_{t=0}=u_{j}(x) \quad \text { in } \mathbf{R}^{n} \quad(0 \leq j \leq m-1)
\end{array}\right.
$$

in the framework of the space of $C^{\infty}$ functions, where $D_{t}=-i \partial / \partial t\left(=-i \partial_{t}\right)$, $D_{x}=\left(D_{1}, \ldots, D_{n}\right)=-i\left(\partial / \partial x_{1}, \ldots, \partial / \partial x_{n}\right), f(t, x) \in C^{\infty}\left([0, \infty) \times \mathbf{R}^{n}\right)$ and $u_{j}(x) \in$ $C^{\infty}\left(\mathbf{R}^{n}\right)(0 \leq j \leq m-1)$.

Definition 1.1. The Cauchy problem $(\mathrm{CP})$ is said to be $C^{\infty}$ well-posed if the following conditions $(\mathrm{E})$ and $(\mathrm{U})$ are satisfied:

(E) For any $f \in C^{\infty}\left([0, \infty) \times \mathbf{R}^{n}\right)$ and $u_{j} \in C^{\infty}\left(\mathbf{R}^{n}\right)(0 \leq j \leq m-1)$ there is $u \in C^{\infty}\left([0, \infty) \times \mathbf{R}^{n}\right)$ satisfying $(\mathrm{CP})$.

(U) If $s>0, u \in C^{\infty}\left([0, \infty) \times \mathbf{R}^{n}\right),\left.\quad D_{t}^{j} u(t, x)\right|_{t=0}=0 \quad(0 \leq j \leq m-1)$ and $P\left(t, D_{t}, D_{x}\right) u(t, x)$ vanishes for $t<s$, then $u(t, x)$ also vanishes for $t<s$.

We assume throughout the paper that

(A-1) $a_{j, \alpha}(t)(1 \leq j \leq m,|\alpha|=j)$ are real analytic on $[0, \infty)$, i.e., the coefficients of the principal part of $P\left(t, D_{t}, D_{x}\right)$ are real analytic on $[0, \infty)$. 
From (A-1) there are a complex neighborhood $\Omega$ of $[0, \infty)$ (in $\mathbf{C}$ ) and $\delta>0$ such that $[-\delta, \infty) \subset \Omega$ and $a_{j, \alpha}(t) \quad(1 \leq j \leq m,|\alpha|=j)$ are regarded as analytic functions defined in $\Omega$. Put

$$
\begin{aligned}
& p(t, \tau, \xi)=\tau^{m}+\sum_{j=1}^{m} a_{j}^{0}(t, \xi) \tau^{m-j} \quad\left(\equiv P_{m}(t, \tau, \xi)\right), \\
& a_{j}^{0}(t, \xi)=\sum_{|\alpha|=j} a_{j, \alpha}(t) \xi^{\alpha}, \\
& P_{k}(t, \tau, \xi)=\sum_{j=m-k|\alpha|=k+j-m}^{m} a_{j, \alpha}(t) \tau^{m-j} \xi^{\alpha} \quad(0 \leq k \leq m-1) .
\end{aligned}
$$

We also assume that

(A-2) $p(t, \tau, \xi)$ is hyperbolic with respect to $\vartheta \equiv(1,0, \ldots, 0) \in \mathbf{R}^{n+1}$ for $t \in$ $[-\delta, \infty)$, i.e.,

$$
p(t, \tau-i, \xi) \neq 0 \text { for any }(t, \tau, \xi) \in[-\delta, \infty) \times \mathbf{R} \times \mathbf{R}^{n} .
$$

Let $\Gamma(p(t, \cdot, \cdot), \vartheta)$ be the connected component of the set $\left\{(\tau, \xi) \in \mathbf{R}^{n+1} \backslash\{0\}\right.$; $p(t, \tau, \xi) \neq 0\}$ which contains $\vartheta$, and define the generalized flows $K_{\left(t_{0}, x^{0}\right)}^{ \pm}$for $p(t, \tau, \xi)$ by

$$
K_{\left(t_{0}, x^{0}\right)}^{ \pm}=\left\{(t(s), x(s)) \in[0, \infty) \times \mathbf{R}^{n} ; \pm s \geq 0 \text { and }\{(t(s), x(s))\}\right. \text { is }
$$

a Lipschitz continuous curve in $[0, \infty) \times \mathbf{R}^{n}$ satisfying

$$
\left.(d / d s)(t(s), x(s)) \in \Gamma(p(t, \cdot, \cdot), \vartheta)^{*}(\text { a.e. } s) \text { and }(t(0), x(0))=\left(t_{0}, x^{0}\right)\right\},
$$

where $\left(t_{0}, x^{0}\right) \in[0, \infty) \times \mathbf{R}^{n} \quad$ and $\quad \Gamma^{*}=\left\{(t, x) \in \mathbf{R}^{n+1} ; t \tau+x \cdot \xi \geq 0\right.$ for any $(\tau, \xi) \in \Gamma\}$. To describe conditions on the lower order terms we define the polynomials $h_{j}(t, \tau, \xi)\left(\equiv h_{j}(t, \tau, \xi ; p)\right)$ of $(\tau, \xi)$ by

$$
|p(t, \tau-i \gamma, \xi)|^{2}=\sum_{j=0}^{m} \gamma^{2 j} h_{m-j}(t, \tau, \xi) \quad \text { for }(t, \tau, \xi) \in[0, \infty) \times \mathbf{R} \times \mathbf{R}^{n} \text { and } \gamma \in \mathbf{R} \text {. }
$$

Since $|p(t, \tau-i \gamma, \xi)|^{2}=\prod_{j=1}^{m}\left(\left(\tau-\lambda_{j}(t, \xi)\right)^{2}+\gamma^{2}\right)$, we have

$$
h_{k}(t, \xi)=\sum_{1 \leq j_{1}<j_{2}<\cdots<j_{k} \leq m} \prod_{l=1}^{k}\left(\tau-\lambda_{j_{l}}(t, \xi)\right)^{2} \quad(1 \leq k \leq m),
$$


where $p(t, \tau, \xi)=\prod_{j=1}^{m}\left(\tau-\lambda_{j}(t, \xi)\right)$. Let $\mathscr{R}(\xi)$ be a set-valued function, whose values are discrete subsets of $[0, \infty)$, defined for $\xi \in S^{n-1}$ satisfying the following:

(i) $\mathscr{R}(\xi) \subset[0, \infty)$ for $\xi \in S^{n-1} \equiv\left\{\xi \in \mathbf{R}^{n} ;|\xi|=1\right\}$.

(ii) For any $T>0$ there is $N_{T} \in \mathbf{Z}_{+}$such that

$$
\#\{\mathscr{R}(\xi) \cap[0, T]\} \leq N_{T} \quad \text { for } \xi \in S^{n-1} .
$$

Here $\# A$ denotes the number of the elements of a set $A$. First we consider the case where the characteristic roots are at most double, i.e.,

(D) If $(t, \tau, \xi) \in[0, \infty) \times \mathbf{R} \times S^{n-1} \quad$ and $\quad p(t, \tau, \xi)=\partial_{\tau} p(t, \tau, \xi)=0$, then $\partial_{\tau}^{2} p(t, \tau, \xi) \neq 0$.

We assume that the following condition (D-L) is satisfied, which is corresponding to a so-called Levi condition:

(D-L) For any $T>0$ there is $C>0$ satisfying

$$
\begin{aligned}
& \min \left\{\min _{s \in \mathscr{R}(\xi)}|t-s|, 1\right\}|\operatorname{sub} \sigma(P)(t, \tau, \xi)| \\
& \quad \leq C h_{m-1}(t, \tau, \xi)^{1 / 2} \text { for }(t, \tau, \xi) \in[0, T] \times \mathbf{R} \times S^{n-1},
\end{aligned}
$$

where $\min _{s \in \mathscr{R}(\xi)}|t-s|=1$ if $\mathscr{R}(\xi)=\varnothing$.

Here $\operatorname{sub} \sigma(P)(t, \tau, \xi)$ denotes the subprincipal symbol of $P\left(t, D_{t}, D_{x}\right)$, i.e.,

$$
\operatorname{sub} \sigma(P)(t, \tau, \xi)=P_{m-1}(t, \tau, \xi)+(i / 2) \partial_{t} \partial_{\tau} p(t, \tau, \xi) .
$$

Then we have the following

THEOREM 1.2. We assume that the conditions (A-1), (A-2), (D) and (D-L) are satisfied. Then the Cauchy problem $(\mathrm{CP})$ is $C^{\infty}$ well-posed. Moreover, if $\left(t_{0}, x^{0}\right)$ $\in(0, \infty) \times \mathbf{R}^{n}$ and $u \in C^{\infty}\left([0, \infty) \times \mathbf{R}^{n}\right)$ satisfies $(\mathrm{CP}), u_{j}(x)=0$ near $\left\{x \in \mathbf{R}^{n}\right.$; $\left.(0, x) \in K_{\left(t_{0}, x^{0}\right)}^{-}\right\} \quad(0 \leq j \leq m-1)$ and $f=0$ near $K_{\left(t_{0}, x^{0}\right)}^{-}\left(\right.$in $\left.[0, \infty) \times \mathbf{R}^{n}\right)$, then $\left(t_{0}, x^{0}\right) \notin \operatorname{supp} u$.

REMARK. The condition (D-L) is necessary for $C^{\infty}$ well-posedness if $p(t, \tau, \xi) \equiv p(\tau, \xi) \quad$ (see [12]). Moreover, (D-L) is the same condition as given in $[16]$ if $m=2$. 
Next we consider the third order case, i.e.,

(T) $m=3$.

We define the sub-sub-principal symbol $\operatorname{sub}^{2} \sigma(P)(t, \tau, \xi)$ of $P\left(t, D_{t}, D_{x}\right)$ by

$$
\begin{aligned}
s u b^{2} \sigma(P)(t, \tau, \xi)= & P_{1}(t, \tau, \xi)+(1 / 6) \partial_{t}^{2} \partial_{\tau}^{2} p(t, \tau, \xi) \\
& +(i / 12) \partial_{\tau}^{2} P_{2}(t, \tau, \xi) \cdot \partial_{t} \partial_{\tau}^{2} p(t, \tau, \xi)
\end{aligned}
$$

and assume that the following condition (T-L) is satisfied:

(T-L) For any $T>0$ there is $C>0$ satisfying

$$
\begin{aligned}
& \min \left\{\min _{s \in \mathscr{R}(\xi)}|t-s|, 1\right\}|s u b \sigma(P)(t, \tau, \xi)| \leq C h_{2}(t, \tau, \xi)^{1 / 2}, \\
& \min \left\{\min _{s \in \mathscr{R}(\xi)}|t-s|^{2}, 1\right\}\left|s u b^{2} \sigma(P)(t, \tau, \xi)\right| \\
& \leq C h_{1}(t, \tau, \xi)^{1 / 2} \text { for }(t, \tau, \xi) \in[0, T] \times \mathbf{R} \times S^{n-1}
\end{aligned}
$$

Now we can state our main result.

THEOREM 1.3. We assume that the conditions (A-1), (A-2), (T) and (T-L) are satisfied. Then the conclusion of Theorem 1.2 also holds.

REMARK. If $p(t, \tau, \xi) \equiv p(\tau, \xi)$, then the condition (T-L) is necessary for $C^{\infty}$ well-posedness (see [12]).

We should note that Colombini-Orrú [1], D’Ancona-Kinoshita [3], ColombiniTaglialatela [2] and Ishida [7] investigated the Cauchy problem for higher-order hyperbolic operators with time-dependent coefficients and gave sufficient conditions for $C^{\infty}$ well-posedness. In their sufficient conditions they also imposed restrictions on

$$
\left(\left|\partial_{t} \lambda_{j}(t, \xi)\right|+\left|\partial_{t} \lambda_{k}(t, \xi)\right|\right) /\left|\lambda_{j}(t, \xi)-\lambda_{k}(t, \xi)\right|
$$

This means that the principal parts of the operators must satisfy some conditions in general. On the other hand, one believes that the Cauchy problem for hyperbolic operators with time-dependent coefficients is $C^{\infty}$ well-posed with suitable choices of the lower order terms if, for example, the coefficients of the principal parts are real analytic. 
The remainder of this paper is organized as follows. In $\S 2$ we shall give preliminary lemmas. Theorem 1.2 will be proved in $\S 3$. Theorem 1.3 will be proved in $\S 4$. In $\S 5$ some remarks and examples will be given.

\section{Preliminaries}

We begin with a simple lemma concerning polynomials with real analytic coefficients.

Lemma 2.1. Let $f(t, \zeta)$ be a polynomial of $\zeta=\left(\zeta_{1}, \ldots, \zeta_{d}\right)$ whose coefficients are real analytic functions of $t$ in $[0, \infty)$. Then, for any $T>0$ there is $N_{T} \in \mathbf{Z}_{+}$ such that

$$
\begin{aligned}
& \sum_{k=0}^{N_{T}}\left|\partial_{t}^{k} f(t, \zeta)\right| \neq 0 \quad \text { for } t \in[0, T], \\
& \#\{t \in[0, T] ; f(t, \zeta)=0\} \leq N_{T}
\end{aligned}
$$

if $\zeta \in \mathbf{R}^{d}$ and $f(t, \zeta) \not \equiv 0$ in $t$.

Proof. Write

$$
f(t, \zeta)=\sum_{|\alpha| \leq m} f_{\alpha}(t) \zeta^{\alpha}
$$

where $m \in \mathbf{N}$ and $\alpha=\left(\alpha_{1}, \ldots, \alpha_{d}\right) \in \mathbf{Z}_{+}^{d}$. We put $L=\#\left\{\alpha \in \mathbf{Z}_{+}^{d} ;|\alpha| \leq m\right\}$, i.e., $L=\left(\begin{array}{c}d+m \\ m\end{array}\right)$, and

$$
F(t, Z)=\sum_{|\alpha| \leq m} f_{\alpha}(t) Z_{\alpha}
$$

where $Z=\left(Z_{\alpha}\right)_{|\alpha| \leq m} \in \mathbf{R}^{L}$. Define

$$
V=\left\{Z \in \mathbf{R}^{L} ; F(t, Z) \equiv 0 \text { in } t\right\} .
$$

Then $V$ is a subspace of $\mathbf{R}^{L}$. So there are $r \in \mathbf{Z}_{+}$and an $L \times L$ non-singular matrix $Q$ such that

$$
V=\left\{\tilde{Z} Q ; \tilde{Z}^{\prime}=0 \in \mathbf{R}^{r}\right\},
$$

where $\tilde{Z}^{\prime}=\left(\tilde{Z}_{1}, \ldots, \tilde{Z}_{r}\right) \in \mathbf{R}^{r}$ for $\tilde{Z}=\left(\tilde{Z}_{1}, \ldots, \tilde{Z}_{L}\right) \in \mathbf{R}^{L}$, and $\tilde{Z}^{\prime}=0$ and $V=\mathbf{R}^{L}$ if $r=0$. Then we can write

$$
F(t, Z)=\tilde{F}\left(t, \tilde{Z}^{\prime}\right)=\sum_{j=1}^{r} \tilde{f}_{j}(t) \tilde{Z}_{j}
$$


where $Z=\tilde{Z} Q$. Note that

$$
\begin{aligned}
& \tilde{F}\left(t, \lambda \tilde{Z}^{\prime}\right)=\lambda \tilde{F}\left(t, \tilde{Z}^{\prime}\right) \text { for } \lambda \in \mathbf{R}, \\
& \tilde{Z}^{\prime} \neq 0 \Leftrightarrow Z(\equiv \tilde{Z} Q) \notin V \Leftrightarrow F(t, Z) \not \equiv 0 \text { in } t .
\end{aligned}
$$

Let $T>0$ and $\left(t_{0}, \tilde{Z}^{0 \prime}\right) \in[0, T] \times S^{r-1}$. From the Weierstrass preparation theorem it follows that there are $\delta_{0}>0$, a neighborhood $U_{0}$ of $\tilde{Z}^{0 \prime}$ in $\mathbf{R}^{r}, \mu_{0} \in \mathbf{Z}_{+}$, a real analytic function $c_{0}\left(t, \tilde{Z}^{\prime}\right)$ defined in $\left[t_{0}-\delta_{0}, t_{0}+\delta_{0}\right] \times \bar{U}_{0}$ and real analytic functions $a_{0, k}\left(\tilde{Z}^{\prime}\right)$ defined in $\bar{U}_{0}\left(1 \leq k \leq \mu_{0}\right)$ such that $c_{0}\left(t, \tilde{Z}^{\prime}\right) \neq 0$,

$$
\tilde{\boldsymbol{F}}\left(t, \tilde{\boldsymbol{Z}}^{\prime}\right)=c_{0}\left(t, \tilde{\boldsymbol{Z}}^{\prime}\right)\left\{\left(t-t_{0}\right)^{\mu_{0}}+a_{0,1}\left(\tilde{\boldsymbol{Z}}^{\prime}\right)\left(t-t_{0}\right)^{\mu_{0}-1}+\cdots+a_{0, \mu_{0}}\left(\tilde{\boldsymbol{Z}}^{\prime}\right)\right\}
$$

for $\left(t, \tilde{Z}^{\prime}\right) \in\left(\left[t_{0}-\delta_{0}, t_{0}+\delta_{0}\right] \cap[0, T]\right) \times \bar{U}_{0}$, and

$$
a_{0, k}\left(\tilde{Z}^{0 \prime}\right)=0 \quad\left(1 \leq k \leq \mu_{0}\right),
$$

where $\tilde{F}\left(t, \tilde{Z}^{\prime}\right)=c_{0}\left(t, \tilde{Z}^{\prime}\right)$ if $\mu_{0}=0$. Then we have

$$
\left.\partial_{t}^{\mu_{0}} \tilde{\boldsymbol{F}}\left(t, \tilde{Z}^{0 \prime}\right)\right|_{t=t_{0}}=\mu_{0} ! c_{0}\left(t_{0}, \tilde{Z}^{0 \prime}\right) \neq 0 .
$$

So we may assume that

$$
\partial_{t}^{\mu_{0}} \tilde{\boldsymbol{F}}\left(t, \tilde{Z}^{\prime}\right) \neq 0 \quad \text { for }\left(t, \tilde{Z}^{\prime}\right) \in\left(\left[t_{0}-\delta_{0}, t_{0}+\delta_{0}\right] \cap[0, T]\right) \times \bar{U}_{0},
$$

modifying $\delta_{0}$ and $U_{0}$ if necessary. Since $[0, T] \times S^{r-1}$ is compact and $\tilde{F}\left(t, \tilde{Z}^{\prime}\right)$ is homogeneous of degree 1 in $\tilde{Z}^{\prime}$, there is $N_{T} \in \mathbf{Z}_{+}$satisfying

$$
\begin{aligned}
& \sum_{k=0}^{N_{T}}\left|\partial_{t}^{k} \tilde{F}\left(t, \tilde{Z}^{\prime}\right)\right| \neq 0 \quad \text { for }\left(t, \tilde{Z}^{\prime}\right) \in[0, T] \times\left(\mathbf{R}^{r} \backslash\{0\}\right), \\
& \#\left\{t \in[0, T] ; \tilde{F}\left(t, \tilde{Z}^{\prime}\right)=0\right\} \leq N_{T} \text { for } \tilde{Z}^{\prime} \in \mathbf{R}^{r} \backslash\{0\}
\end{aligned}
$$

Put

$$
\begin{aligned}
& Z(\zeta)=\left(\zeta^{\alpha}\right)_{|\alpha| \leq m}, \quad \tilde{Z}(\zeta) \equiv\left(\tilde{Z}_{1}(\zeta), \ldots, \tilde{Z}_{L}(\zeta)\right)=Z(\zeta) Q^{-1} \\
& \tilde{Z}^{\prime}(\zeta)=\left(\tilde{Z}_{1}(\zeta), \ldots, \tilde{Z}_{r}(\zeta)\right)
\end{aligned}
$$

Since $f(t, \zeta)=\tilde{F}\left(t, \tilde{Z}^{\prime}(\zeta)\right)$, and $\tilde{Z}^{\prime}(\zeta) \neq 0$ if $f(t, \zeta) \not \equiv 0$ in $t,(2.1)$ and (2.2) prove the lemma. 
Write

$$
\begin{aligned}
& p(\tau)=\prod_{j=1}^{m}\left(\tau-\lambda_{j}\right), \\
& p_{\varepsilon}(\tau)=(1+\varepsilon(d / d \tau)) p(\tau), \\
& |p(\tau-i \gamma)|^{2}=\sum_{j=0}^{m} \gamma^{2 j} h_{m-j}(\tau ; p) \quad \text { for } \tau \in \mathbf{R} \text { and } \gamma \in \mathbf{R},
\end{aligned}
$$

where $\lambda_{j} \in \mathbf{R}(1 \leq j \leq m)$ and $\varepsilon \in \mathbf{R}$. Then we have the following

Lemma 2.2. For $\tau \in \mathbf{R}, \varepsilon \in \mathbf{R}$ and $1 \leq j \leq m$ we have

$$
h_{m-j}\left(\tau ; p_{\varepsilon}\right) \geq\left\{3\left(\begin{array}{c}
m \\
j-1
\end{array}\right)\right\}^{-1} h_{m-j}(\tau ; p) .
$$

Proof. Lemma 2.1 of Svensson [11] gives

$$
\begin{aligned}
& (m-r) !(k-r) ! /(m ! k !) \\
& \quad \leq h_{m-k}(\tau ; p) / h_{m-k}\left(\tau ; p^{(r)}\right) \\
& \quad \leq(k-1-r) !(k-r) ! /((k-1) ! k !) \quad(0 \leq r<k \leq m),
\end{aligned}
$$

where $p^{(r)}(\tau)=\partial_{\tau}^{r} p(\tau)$. In particular, we have

$$
\begin{aligned}
(m-r) ! /(m !(r+1) !) & \leq h_{m-r-1}(\tau ; p) / h_{m-r-1}\left(\tau ; p^{(r)}\right) \\
& \leq 1 /(r !(r+1) !) \quad(0 \leq r<m) .
\end{aligned}
$$

Therefore, it suffices to show that

$$
h_{m-1}\left(\tau ; p_{\varepsilon}\right) \geq h_{m-1}(\tau ; p) / 3 \text { for } \tau \in \mathbf{R} \text { and } \varepsilon \in \mathbf{R},
$$

in order to prove the lemma. Indeed, (2.4) and (2.5) with $p$ replaced by $p^{(r)}$ yield

$$
\begin{aligned}
h_{m-r-1}\left(\tau ; p_{\varepsilon}\right) & \geq(m-r) ! h_{m-r-1}\left(\tau ; p_{\varepsilon}^{(r)}\right) /(m !(r+1) !) \\
& \geq(m-r) ! h_{m-r-1}\left(\tau ; p^{(r)}\right) /(3 m !(r+1) !) \\
& \geq(m-r) ! r ! h_{m-r-1}(\tau ; p) /(3 m !)
\end{aligned}
$$


$(0 \leq r<m)$. Put $\sigma_{j}=\tau-\lambda_{j}$. Since

$$
-\lim _{\gamma \downarrow 0} \operatorname{Im}\left\{p(\tau-i \gamma) \cdot p^{(1)}(\tau+i \gamma)\right\} / \gamma=\sum_{j=1}^{m} \prod_{k \neq j} \sigma_{k}^{2}=h_{m-1}(\tau ; p),
$$

a simple calculation yields

$$
\begin{aligned}
h_{m-1}\left(\tau ; p_{\varepsilon}\right)=-\lim _{\gamma \downarrow 0} \operatorname{Im}\{ & \left(p(\tau-i \gamma)+\varepsilon p^{(1)}(\tau-i \gamma)\right) \\
& \left.\times\left(p^{(1)}(\tau+i \gamma)+\varepsilon p^{(2)}(\tau+i \gamma)\right)\right\} / \gamma \\
= & \sum_{1 \leq j<k<l \leq m}\left(\prod_{\substack{1 \leq \mu \leq m, \mu \neq j, k, l}} \sigma_{\mu}^{2}\right) I_{j, k, l}^{\varepsilon},
\end{aligned}
$$

where

$$
\begin{aligned}
I_{j, k, l}^{\varepsilon}= & 2 \varepsilon^{2}\left\{2 \sigma_{j}^{2}+2 \sigma_{k}^{2}+2 \sigma_{l}^{2}+\sigma_{j} \sigma_{k}+\sigma_{j} \sigma_{l}+\sigma_{k} \sigma_{l}\right\} \\
& +2 \varepsilon\left\{\sigma_{j}^{2} \sigma_{k}+\sigma_{j}^{2} \sigma_{l}+\sigma_{j} \sigma_{k}^{2}+\sigma_{k}^{2} \sigma_{l}+\sigma_{j} \sigma_{l}^{2}+\sigma_{k} \sigma_{l}^{2}\right\} \\
& +\sigma_{j}^{2} \sigma_{k}^{2}+\sigma_{j}^{2} \sigma_{l}^{2}+\sigma_{k}^{2} \sigma_{l}^{2} .
\end{aligned}
$$

Indeed, for example, we have

$$
\begin{aligned}
-\lim _{\gamma \downarrow 0} \operatorname{Im}\left\{p^{(1)}(\tau-i \gamma) p^{(2)}(\tau+i \gamma)\right\} / \gamma \\
=-\lim _{\gamma \downarrow 0} 2 \operatorname{Im}\left\{\sum_{j=1}^{m} \prod_{v \neq j}\left(\sigma_{v}-i \gamma\right) \cdot \sum_{1 \leq k<l \leq m} \prod_{\mu \neq k, l}\left(\sigma_{\mu}+i \gamma\right)\right\} / \gamma \\
=2\left\{\sum_{1 \leq j<k<l \leq m}+\sum_{1 \leq k<j<l \leq m}+\sum_{1 \leq k<l<j \leq m}\right\}\left(-\sigma_{k} \sigma_{l}+\sigma_{j} \sigma_{k}+\sigma_{j} \sigma_{l}\right) \prod_{\mu \neq j, k, l} \sigma_{\mu}^{2} \\
+4 \sum_{1 \leq k<l \leq m} \prod_{\mu \neq k, l} \sigma_{\mu}^{2} \\
=2 \sum_{1 \leq j<k<l \leq m}\left(\left(\sigma_{j} \sigma_{k}+\sigma_{j} \sigma_{l}+\sigma_{k} \sigma_{l}\right)+2\left(\sigma_{j}^{2}+\sigma_{k}^{2}+\sigma_{l}^{2}\right)\right) \prod_{\mu \neq j, k, l} \sigma_{\mu}^{2}
\end{aligned}
$$

Let $1 \leq j<k<l \leq m$, and put

$$
\begin{aligned}
& \Xi_{j, k, l}=3\left(\sigma_{j}^{2}+\sigma_{k}^{2}+\sigma_{l}^{2}\right)+\left(\sigma_{j}+\sigma_{k}+\sigma_{l}\right)^{2} \\
& \left(=2\left\{2 \sigma_{j}^{2}+2 \sigma_{k}^{2}+2 \sigma_{l}^{2}+\sigma_{j} \sigma_{k}+\sigma_{j} \sigma_{l}+\sigma_{k} \sigma_{l}\right\}\right) .
\end{aligned}
$$


If $\Xi_{j, k, l}=0$, then $\sigma_{j}=\sigma_{k}=\sigma_{l}=0$. Therefore, we have

$$
(0=) 3 I_{j, k, l}^{\varepsilon} \geq \sigma_{j}^{2} \sigma_{k}^{2}+\sigma_{j}^{2} \sigma_{l}^{2}+\sigma_{k}^{2} \sigma_{l}^{2}(=0) \text { if } \Xi_{j, k, l}=0 .
$$

Now assume that $\Xi_{j, k, l} \neq 0$. Then we have

$$
\begin{aligned}
I_{j, k, l}^{\varepsilon}= & \Xi_{j, k, l}\left\{\varepsilon+\left(\sigma_{j}^{2}\left(\sigma_{k}+\sigma_{l}\right)+\sigma_{k}^{2}\left(\sigma_{j}+\sigma_{l}\right)+\sigma_{l}^{2}\left(\sigma_{j}+\sigma_{k}\right)\right) / \Xi_{j, k, l}\right\}^{2} \\
& +J_{j, k, l} / \Xi_{j, k, l} \geq J_{j, k, l} / \Xi_{j, k, l}
\end{aligned}
$$

where

$$
\begin{aligned}
J_{j, k, l}= & -\left\{\sigma_{j}^{2}\left(\sigma_{k}+\sigma_{l}\right)+\sigma_{k}^{2}\left(\sigma_{j}+\sigma_{l}\right)+\sigma_{l}^{2}\left(\sigma_{j}+\sigma_{k}\right)\right\}^{2} \\
& +\Xi_{j, k, l}\left(\sigma_{j}^{2} \sigma_{k}^{2}+\sigma_{j}^{2} \sigma_{l}^{2}+\sigma_{k}^{2} \sigma_{l}^{2}\right) .
\end{aligned}
$$

A simple calculation yields

$$
\begin{aligned}
J_{j, k, l}= & \sigma_{j}^{4}\left(\sigma_{k}-\sigma_{l}\right)^{2}+\sigma_{k}^{4}\left(\sigma_{j}-\sigma_{l}\right)^{2}+\sigma_{l}^{4}\left(\sigma j-\sigma_{l}\right)^{2} \\
& +2 \sigma_{j}^{4}\left(\sigma_{k}^{2}+\sigma_{l}^{2}\right)+2 \sigma_{k}^{4}\left(\sigma_{j}^{2}+\sigma_{l}^{2}\right)+2 \sigma_{l}^{4}\left(\sigma_{j}^{2}+\sigma_{k}^{2}\right)+6 \sigma_{j}^{2} \sigma_{k}^{2} \sigma_{l}^{2} \\
\geq & 2 \sigma_{j}^{4}\left(\sigma_{k}^{2}+\sigma_{l}^{2}\right)+2 \sigma_{k}^{4}\left(\sigma_{j}^{2}+\sigma_{l}^{2}\right)+2 \sigma_{l}^{4}\left(\sigma_{j}^{2}+\sigma_{k}^{2}\right)+6 \sigma_{j}^{2} \sigma_{k}^{2} \sigma_{l}^{2} .
\end{aligned}
$$

This yields

$$
\begin{aligned}
& \Xi_{j, k, l}\left(\sigma_{j}^{2} \sigma_{k}^{2}+\sigma_{j}^{2} \sigma_{l}^{2}+\sigma_{k}^{2} \sigma_{l}^{2}\right) \\
&=4 \sigma_{j}^{4}\left(\sigma_{k}^{2}+\sigma_{l}^{2}\right)+4 \sigma_{k}^{4}\left(\sigma_{j}^{2}+\sigma_{l}^{2}\right)+4 \sigma_{l}^{4}\left(\sigma_{j}^{2}+\sigma_{k}^{2}\right)+12 \sigma_{j}^{2} \sigma_{k}^{2} \sigma_{l}^{2} \\
&+2 \sigma_{j} \sigma_{k}^{3} \sigma_{l}^{2}+2 \sigma_{j} \sigma_{k}^{2} \sigma_{l}^{3}+2 \sigma_{j}^{3} \sigma_{k} \sigma_{l}^{2}+2 \sigma_{j}^{2} \sigma_{k} \sigma_{l}^{3}+2 \sigma_{j}^{3} \sigma_{k}^{2} \sigma_{l} \\
&+2 \sigma_{j}^{2} \sigma_{k}^{3} \sigma_{l}+2 \sigma_{j}^{3} \sigma_{k}^{3}+2 \sigma_{j}^{3} \sigma_{l}^{3}+2 \sigma_{k}^{3} \sigma_{l}^{3} \\
& \leq 4 \sigma_{j}^{4}\left(\sigma_{k}^{2}+\sigma_{l}^{2}\right)+4 \sigma_{k}^{4}\left(\sigma_{j}^{2}+\sigma_{l}^{2}\right)+4 \sigma_{l}^{4}\left(\sigma_{j}^{2}+\sigma_{k}^{2}\right)+12 \sigma_{j}^{2} \sigma_{k}^{2} \sigma_{l}^{2} \\
&+ \sigma_{k}^{2} \sigma_{l}^{2}\left(\sigma_{j}^{2}+\sigma_{k}^{2}\right)+\sigma_{k}^{2} \sigma_{l}^{2}\left(\sigma_{j}^{2}+\sigma_{l}^{2}\right)+\sigma_{j}^{2} \sigma_{l}^{2}\left(\sigma_{j}^{2}+\sigma_{k}^{2}\right) \\
&+\sigma_{j}^{2} \sigma_{l}^{2}\left(\sigma_{k}^{2}+\sigma_{l}^{2}\right)+\sigma_{j}^{2} \sigma_{k}^{2}\left(\sigma_{j}^{2}+\sigma_{l}^{2}\right)+\sigma_{j}^{2} \sigma_{k}^{2}\left(\sigma_{k}^{2}+\sigma_{l}^{2}\right) \\
&+\sigma_{k}^{2} \sigma_{l}^{2}\left(\sigma_{k}^{2}+\sigma_{l}^{2}\right)+\sigma_{j}^{2} \sigma_{l}^{2}\left(\sigma_{j}^{2}+\sigma_{l}^{2}\right)+\sigma_{j}^{2} \sigma_{k}^{2}\left(\sigma_{j}^{2}+\sigma_{k}^{2}\right) \\
&= 3\left(2 \sigma_{j}^{4}\left(\sigma_{k}^{2}+\sigma_{l}^{2}\right)+2 \sigma_{k}^{4}\left(\sigma_{j}^{2}+\sigma_{l}^{2}\right)+2 \sigma_{l}^{4}\left(\sigma_{j}^{2}+\sigma_{k}^{2}\right)+6 \sigma_{j}^{2} \sigma_{k}^{2} \sigma_{l}^{2}\right) \\
& \leq 3 J_{j, k, l},
\end{aligned}
$$


since $2 \sigma_{j} \sigma_{k} \leq \sigma_{j}^{2}+\sigma_{k}^{2}, \cdots$. Therefore, from (2.6) and (2.7) we have

$$
\begin{aligned}
h_{m-1}\left(\tau ; p_{\varepsilon}\right) & \geq \sum_{1 \leq j<k<l \leq m}\left(\prod_{\substack{1 \leq \mu \leq m, j \\
\mu \neq j, k, l}} \sigma_{\mu}^{2}\right)\left(\sigma_{j}^{2} \sigma_{k}^{2}+\sigma_{j}^{2} \sigma_{l}^{2}+\sigma_{k}^{2} \sigma_{l}^{2}\right) / 3 \\
& =h_{m-1}(\tau ; p) / 3,
\end{aligned}
$$

which proves (2.5) and the lemma.

Now we assume that (A-1) and (A-2) are satisfied, and define

$$
p_{\varepsilon}(t, \tau, \xi)=\left(1-\varepsilon^{2}|\xi|^{2} \partial_{\tau}^{2}\right) p(t, \tau, \xi)
$$

for $\varepsilon \in \mathbf{R},(t, \tau, \xi) \in[0, \infty) \times \mathbf{R} \times \mathbf{R}^{n}$, changing the notation. We note that $p_{\varepsilon}(t, \tau, \xi)$ is strictly hyperbolic with respect to $\vartheta$ for $\varepsilon \in \mathbf{R} \backslash\{0\}$ if $p(t, \tau, \xi)$ has at most triple characteristics (see [10]). Lemma 2.2 gives

$$
h_{m-j}\left(t, \tau, \xi ; p_{\varepsilon}\right) \geq\left\{3\left(\begin{array}{c}
m \\
j-1
\end{array}\right)\right\}^{-2} h_{m-j}(t, \tau, \xi ; p)
$$

for $1 \leq j \leq m, \varepsilon \in \mathbf{R},(t, \tau, \xi) \in[0, \infty) \times \mathbf{R} \times \mathbf{R}^{n}$. We note that one can directly prove that

$$
h_{j}\left(t, \tau, \xi ; p_{\varepsilon}\right) \geq h_{j}(t, \tau, \xi ; p) \quad(0 \leq j \leq 2)
$$

if $m=3$. Write

$$
p_{\varepsilon}(t, \tau, \xi)=\prod_{j=1}^{m}\left(\tau-\lambda_{j}(t, \xi ; \varepsilon)\right) .
$$

Lemma 2.3. For each fixed $\xi \in S^{n-1}$ and $\varepsilon \in \mathbf{R}$ we can enumerate $\left\{\lambda_{j}(t, \xi ; \varepsilon)\right\}$ so that the $\lambda_{j}(t, \xi ; \varepsilon)$ are real analytic in $t \in[0, \infty)$. Moreover, for any $v \in \mathbf{Z}_{+}$there are $\mathscr{N}_{v}^{0}\left(\equiv \mathscr{N}_{v}^{0}(p)\right) \subset \mathbf{R}$ and $\mathscr{N}_{v}(\varepsilon)\left(\equiv \mathscr{N}_{v}(\varepsilon ; p)\right) \subset \mathbf{R}^{n} \backslash\{0\}$ for $\varepsilon \in \mathbf{R} \backslash \mathscr{N}_{v}^{0}$ satisfying the following:

(i) $\lambda \xi \in \mathscr{N}_{v}(\varepsilon)$ if $\lambda>0$ and $\xi \in \mathscr{N}_{v}(\varepsilon)$.

(ii) $\mu_{1}\left(\mathscr{N}_{v}^{0}\right)=0$.

(iii) $\mu_{n}\left(\mathscr{N}_{v}(\varepsilon)\right)=0$ for $\varepsilon \in \mathbf{R} \backslash \mathscr{N}_{v}^{0}$.

(iv) For any $T>0$ there is $N_{T, v} \in \mathbf{Z}_{+}$such that 


$$
\begin{gathered}
\#\left\{t \in[0, T] ; \partial_{t}^{v}\left(\lambda_{j}(t, \xi ; \varepsilon)-\lambda_{k}(t, \xi ; \varepsilon)\right)=0\right\} \leq N_{T, v} \\
\quad \text { if } 1 \leq j<k \leq m \text { and } \partial_{t}^{v}\left(\lambda_{j}(t, \xi ; \varepsilon)-\lambda_{k}(t, \xi ; \varepsilon)\right) \not \equiv 0 \text { in } t, \\
\#\left\{t \in[0, T] ; \partial_{t}^{v} \lambda_{j}(t, \xi ; \varepsilon)=0\right\} \leq N_{T, v} \\
\quad \text { if } 1 \leq j \leq m \text { and } \partial_{t}^{v} \lambda_{j}(t, \xi ; \varepsilon) \not \equiv 0 \text { in } t \\
\text { for } \varepsilon \in \mathbf{R} \backslash \mathscr{N}_{v}^{0} \text { and } \xi \in S^{n-1} \backslash \mathscr{N}_{v}(\varepsilon) .
\end{gathered}
$$

Here $\mu_{n}$ denotes the Lebesgue measure in $\mathbf{R}^{n}$.

REMARK. (i) The $\lambda_{j}(t, \xi ; \varepsilon)$ in the lemma are not necessarily continuous in $(\xi, \varepsilon)$. (ii) If the conditions (D) or (T) are satisfied, then $p_{\varepsilon}(t, \tau, \xi)$ is strictly hyperbolic for $\varepsilon \neq 0$, and the assertion of the first part of the lemma is obvious for $\varepsilon \neq 0$.

Proof. First fix $(\xi, \varepsilon) \in S^{n-1} \times \mathbf{R}$. To simplify the notations we write $p(t, \tau)$ $=p_{\varepsilon}(t, \tau, \xi)$. For $t_{0} \in[0, \infty) \mathscr{A}_{t_{0}}$ denotes the convergent power series ring of $\left(t-t_{0}\right)$. Since $\mathscr{A}_{t_{0}}$ is a unique factorization domain, $\mathscr{A}_{t_{0}}[\tau]$ is also a unique factorization domain. Therefore, we can write

$$
p(t, \tau)=p_{1}(t, \tau)^{r_{1}} p_{2}(t, \tau)^{r_{2}} \cdots p_{\sigma}(t, \tau)^{r_{\sigma}},
$$

where $\sigma, r_{j} \in \mathbf{N}$, the $p_{j}(t, \tau)\left(\in \mathscr{A}_{t_{0}}[\tau]\right)$ are irreducible in $\mathscr{A}_{t_{0}}[\tau]$, and $p_{j}(t, \tau)$ and $p_{k}(t, \tau)$ are mutually prime if $j \neq k$. Since the leading coefficient of $p(t, \tau)$ is equal to 1 , we may assume that the leading coefficients of the $p_{j}(t, \tau)$ are also equal to 1 . Put

$$
q(t, \tau)=\prod_{j=1}^{\sigma} p_{j}(t, \tau) .
$$

We denote by $D(t)$ the discriminant of $q(t, \tau)=0$ in $\tau$. Then we have $D(t) \not \equiv 0$. Indeed, suppose that $D(t) \equiv 0$. Then $q(t, \tau)$ and $\partial_{\tau} q(t, \tau)$ are not mutually prime as polynomials in $\mathscr{A}_{t_{0}}[\tau]$ (see, e.g., Chap. 5 of [5] and $\S$ A.1 of [6]). This leads a contradiction. When $D\left(t_{0}\right) \neq 0, q(t, \tau)$ is strictly hyperbolic in $\tau$ near $t=t_{0}$ and, therefore, we may assume that the $\lambda_{j}(t, \xi ; \varepsilon)$ are analytic in a complex neighborhood of $t_{0}$. Next assume that $D\left(t_{0}\right)=0$. Since the zeros of $D(t)$ are discrete, the $\lambda_{j}(t, \xi ; \varepsilon)$ are analytic in a complex neighborhood of $t_{0}$ except for $t_{0}$. Fix $j_{0}$ so that $1 \leq j_{0} \leq m$. Analytic continuations of $\lambda_{j_{0}}(t, \xi ; \varepsilon)$ around $t_{0}$ and Riemann's theorem on removable singularities show that there is $r \in \mathbf{N}$ such that $\lambda_{j_{0}}\left(t_{0}+z^{r}, \xi ; \varepsilon\right)$ is analytic in a complex neighborhood of $z=0$. Hyperbolicity 
implies that $\lambda_{j_{0}}\left(t_{0}+z^{r}, \xi ; \varepsilon\right)$ is real if $z^{r}$ is real, and that one can take $r=1$, i.e., $\lambda_{j_{0}}(t, \xi ; \varepsilon)$ is analytic in $t$ near $t_{0}$. Starting from $t=0$ and continuing analytically along $[0, \infty)$, we can enumerate $\left\{\lambda_{j}(t, \xi ; \varepsilon)\right\}$ so that the $\lambda_{j}(t, \xi ; \varepsilon)$ are real analytic. This proves the first part of the assertions of the lemma. Next let us prove the second part. Let $\mathscr{A}$ be the ring of the real analytic functions of $t$ defined in $[0, \infty)$. Note that $\mathscr{A}$ is an integral domain and that $\mathscr{A}$ is not a unique factorization domain. We denote by $\Sigma$ the quotient field of $\mathscr{A}[\xi, \varepsilon]$. Then $\Sigma[\tau]$ is a unique factorization domain and $p_{\varepsilon}(t, \tau, \xi) \in \Sigma[\tau]$. Write

$$
\tau p_{\varepsilon}(t, \tau, \xi)=p_{\varepsilon}^{1}(t, \tau, \xi)^{r_{1}} \cdots p_{\varepsilon}^{\sigma}(t, \tau, \xi)^{r_{\sigma}}
$$

where $\sigma, r_{j} \in \mathbf{N}$, the $p_{\varepsilon}^{j}(t, \tau, \xi)(\in \Sigma[\tau])$ are irreducible in $\Sigma[\tau]$ and $p_{\varepsilon}^{j}(t, \tau, \xi)$ and $p_{\varepsilon}^{k}(t, \tau, \xi)$ are mutually prime if $j \neq k$. Here $\sigma$ and the $r_{j}$ are different from those as appeared in (2.9), in general. Define $q(t, \tau, \xi ; \varepsilon)=\prod_{j=1}^{\sigma} p_{\varepsilon}^{j}(t, \tau, \xi)$, and let $D(t, \xi ; \varepsilon)$ be the discriminant of $q(t, \tau, \xi ; \varepsilon)=0$ in $\tau$. We note that

$$
\{\tau \in \mathbf{C} ; q(t, \tau, \xi ; \varepsilon)=0\}=\left\{\tau \in \mathbf{C} ; p_{\varepsilon}(t, \tau, \xi)=0\right\} \cup\{0\} .
$$

Write

$$
D(t, \xi ; \varepsilon)=d_{0}(t, \xi ; \varepsilon) / d_{1}(t, \xi ; \varepsilon),
$$

where $d_{k}(t, \xi ; \varepsilon) \in \mathscr{A}[\xi, \varepsilon]$ and $d_{k}(t, \xi ; \varepsilon) \neq 0$ in $\mathscr{A}[\xi, \varepsilon]$, i.e., $d_{k}(t, \xi ; \varepsilon) \not \equiv 0$ in $(t, \xi, \varepsilon)$ $(k=0,1)$. We may assume that the $d_{k}(t, \xi ; \varepsilon)$ are homogeneous in $\xi$. Indeed, assume that $a_{k}(\xi)(k=0,1)$ are polynomials of $\xi$ and $a_{0}(\xi) / a_{1}(\xi)$ is homogeneous in $\xi$. Write $a_{k}(\xi)=a_{k}^{0}(\xi)+\left(a_{k}(\xi)-a_{k}^{0}(\xi)\right)(k=0,1)$, where $a_{k}^{0}(\xi)$ is the principal part of $a_{k}(\xi)$. Then we have, with some $\kappa \in \mathbf{Z}$,

$$
a_{0}(\xi) / a_{1}(\xi)=\lambda^{-\kappa} a_{0}(\lambda \xi) / a_{1}(\lambda \xi) \rightarrow a_{0}^{0}(\xi) / a_{1}^{0}(\xi) \quad(\lambda \rightarrow \infty)
$$

for $\xi$ with $a_{1}^{0}(\xi) \neq 0$ and, therefore, $a_{0}(\xi) / a_{1}(\xi)=a_{0}^{0}(\xi) / a_{1}^{0}(\xi)$. So we may assume that the $a_{k}(\xi)$ are homogeneous in $\xi$. Put

$$
\tilde{\mathscr{N}}_{0}=\left\{(\xi, \varepsilon) \in\left(\mathbf{R}^{n} \backslash\{0\}\right) \times \mathbf{R} ; d_{0}(t, \xi ; \varepsilon) d_{1}(t, \xi ; \varepsilon) \equiv 0 \text { in } t(\in[0, \infty))\right\} .
$$

Then we have $\mu_{n+1}\left(\tilde{\mathscr{N}}_{0}\right)=0$, since

$$
\mu_{n+2}\left(\left\{(t, \xi, \varepsilon) \in[0, \infty) \times\left(\mathbf{R}^{n} \backslash\{0\}\right) \times \mathbf{R} ; d_{0}(t, \xi ; \varepsilon) d_{1}(t, \xi ; \varepsilon)=0\right\}\right)=0 .
$$

Define

$$
\mathscr{N}_{0}^{0}=\left\{\varepsilon \in \mathbf{R} ; \mu_{n}\left(\left\{\xi \in \mathbf{R}^{n} \backslash\{0\} ;(\xi, \varepsilon) \in \tilde{\mathscr{N}}_{0}\right\}\right)>0\right\} .
$$

Then it is obvious that $\mu_{1}\left(\mathscr{N}_{0}^{0}\right)=0$. For $\varepsilon \in \mathbf{R} \backslash \mathscr{N}_{0}^{0}$ we define

$$
\mathscr{N}_{0}(\varepsilon)=\left\{\xi \in \mathbf{R}^{n} \backslash\{0\} ;(\xi, \varepsilon) \in \tilde{\mathscr{N}}_{0}\right\} .
$$


By definition we see that $\mu_{n}\left(\mathscr{N}_{0}(\varepsilon)\right)=0$ for $\varepsilon \in \mathbf{R} \backslash \mathscr{N}_{0}^{0}$ and that $d_{0}(t, \xi ; \varepsilon) \times$ $d_{1}(t, \xi ; \varepsilon) \not \equiv 0$ in $t$, i.e., $D(t, \xi ; \varepsilon) \not \equiv 0$ in $t$, for $\varepsilon \in \mathbf{R} \backslash \mathscr{N}_{0}^{0}$ and $\xi \in S^{n-1} \backslash \mathscr{N}_{0}(\varepsilon)$. Since the $d_{k}(t, \xi ; \varepsilon)$ are homogeneous in $\xi, \mathscr{N}_{0}(\varepsilon)$ is a cone, i.e., $\lambda \xi \in \mathscr{N}_{0}(\varepsilon)$ if $\lambda>0$ and $\xi \in \mathscr{N}_{0}(\varepsilon)$. For fixed $\varepsilon \in \mathbf{R} \backslash \mathscr{N}_{0}^{0}$ and $\xi \in S^{n-1} \backslash \mathscr{N}_{0}(\varepsilon)$ the roots of $q(t, \tau, \xi ; \varepsilon)=0$ in $\tau$ are simple if $t \in D_{(\xi, \varepsilon)}$, where $D_{(\xi, \varepsilon)}=\left\{t \in[0, \infty) ; d_{0}(t, \xi ; \varepsilon) d_{1}(t, \xi ; \varepsilon) \neq 0\right\}$. So, enumerating $\left\{\lambda_{j}(t, \xi ; \varepsilon)\right\}$, we can write

$$
q(t, \tau, \xi ; \varepsilon)=\prod_{j=1}^{\hat{m}}\left(\tau-\lambda_{j}(t, \xi ; \varepsilon)\right) \quad \text { for } t \in[0, \infty), \varepsilon \in \mathbf{R} \backslash \mathscr{N}_{0}^{0} \text { and } \xi \in S^{n-1} \backslash \mathscr{N}_{0}(\varepsilon),
$$

where $\hat{m}=\operatorname{deg}_{\tau} q(t, \tau, \xi ; \varepsilon)$ and the $\lambda_{j}(t, \xi ; \varepsilon)$ are real analytic in $t \in[0, \infty)$ for $\varepsilon \in \mathbf{R} \backslash \mathscr{N}_{0}^{0}$ and $\xi \in S^{n-1} \backslash \mathscr{N}_{0}(\varepsilon)$. We may assume that $\lambda_{\hat{m}}(t, \xi ; \varepsilon) \equiv 0$. Write

$$
q(t, \tau, \xi ; \varepsilon)=\tau^{\hat{m}}+a_{1}(t, \xi ; \varepsilon) \tau^{\hat{m}-1}+\cdots+a_{\hat{m}-1}(t, \xi ; \varepsilon) \tau,
$$

where $a_{j}(t, \xi ; \varepsilon) \in \Sigma$. Note that the $a_{j}(t, \xi ; \varepsilon)$ are real analytic in $t \in[0, \infty)$ for $\varepsilon \in \mathbf{R} \backslash \mathscr{N}_{0}^{0}$ and $\xi \in S^{n-1} \backslash \mathscr{N}_{0}(\varepsilon)$. Let $\varepsilon \in \mathbf{R} \backslash \mathscr{N}_{0}^{0}$ and $\xi \in S^{n-1} \backslash \mathscr{N}_{0}(\varepsilon)$. Then we have

$$
\left.\partial_{\tau} q(t, \tau, \xi ; \varepsilon)\right|_{\tau=\lambda_{j}(t, \xi ; \varepsilon)} \cdot \partial_{t} \lambda_{j}(t, \xi ; \varepsilon)+\left.\partial_{t} q(t, \tau, \xi ; \varepsilon)\right|_{\tau=\lambda_{j}(t, \xi ; \varepsilon)}=0
$$

for $1 \leq j \leq \hat{m}$. So, for $t \in D_{(\xi, \varepsilon)}$ we have $\left.\partial_{\tau} q(t, \tau, \xi ; \varepsilon)\right|_{\tau=\lambda_{j}(t, \xi ; \varepsilon)} \neq 0$ and

$$
\partial_{t} \lambda_{j}(t, \xi ; \varepsilon)=-\left.\partial_{t} q(t, \tau, \xi ; \varepsilon)\right|_{\tau=\lambda_{j}(t, \xi ; \varepsilon)} /\left.\partial_{\tau} q(t, \tau, \xi ; \varepsilon)\right|_{\tau=\lambda_{j}(t, \xi ; \varepsilon)}
$$

for $1 \leq j \leq \hat{m}$. Since $\lambda_{\hat{m}}(t, \xi ; \varepsilon) \equiv 0$, we have

$$
\prod_{j=1}^{\hat{m}} \partial_{t} \lambda_{j}(t, \xi ; \varepsilon) \equiv 0
$$

Noting that $\prod_{1 \leq j, k \leq \hat{m}, j \neq k}\left(\lambda_{j}(t, \xi ; \varepsilon)-\lambda_{k}(t, \xi ; \varepsilon)\right)=(-1)^{\hat{m}(\hat{m}-1) / 2} D(t, \xi ; \varepsilon)$, we can write the other fundamental symmetric expressions as follows;

$$
\begin{aligned}
& \sum_{j=1}^{\hat{m}} \prod_{k \neq j} \partial_{t} \lambda_{k}(t, \xi ; \varepsilon) \\
& =(-1)^{\hat{m}-1+\hat{m}(\hat{m}-1) / 2} \\
& \quad \times \sum_{j=1}^{\hat{m}} \prod_{k \neq j}\left\{\left.\left(\lambda_{k}(t, \xi ; \varepsilon)-\lambda_{j}(t, \xi ; \varepsilon)\right) \partial_{t} q(t, \tau, \xi ; \varepsilon)\right|_{\tau=\lambda_{k}(t, \xi ; \varepsilon)}\right\} / D(t, \xi ; \varepsilon) \\
& =E_{\hat{m}-1}(t, \xi ; \varepsilon) / D(t, \xi ; \varepsilon), \\
& \quad \ldots \\
& \sum_{j=1}^{\hat{m}} \partial_{t} \lambda_{j}(t, \xi ; \varepsilon)=E_{1}(t, \xi ; \varepsilon) / D(t, \xi ; \varepsilon),
\end{aligned}
$$


where the $E_{k}(t, \xi ; \varepsilon)$ are polynomials of $\left\{a_{j}(t, \xi ; \varepsilon)\right\}_{1 \leq j \leq \hat{m}}$ and $\left\{\partial_{t} a_{j}(t, \xi ; \varepsilon)\right\}_{1 \leq j \leq \hat{m}}$. Put

$$
\begin{aligned}
& \tilde{p}(t, \tau, \xi ; \varepsilon)=\tau^{\hat{m}}-E_{1}(t, \xi ; \varepsilon) D(t, \xi ; \varepsilon)^{-1} \tau^{\hat{m}-1} \\
& \quad+E_{2}(t, \xi ; \varepsilon) D(t, \xi ; \varepsilon)^{-1} \tau^{\hat{m}-2}+\cdots+(-1)^{\hat{m}-1} E_{\hat{m}-1}(t, \xi ; \varepsilon) D(t, \xi ; \varepsilon)^{-1} \tau \\
& \left(=\prod_{j=1}^{\hat{m}}\left(\tau-\partial_{t} \lambda_{j}(t, \xi ; \varepsilon)\right)\right) .
\end{aligned}
$$

Let us repeat the above argument with $\tau p_{\varepsilon}$ replaced by $\tilde{p}$. We write

$$
\tilde{p}(t, \tau, \xi ; \varepsilon)=\tilde{p}^{1}(t, \tau, \xi ; \varepsilon)^{r_{1}^{\prime}} \cdots \tilde{p}^{\sigma^{\prime}}(t, \tau, \xi ; \varepsilon)^{r_{\sigma^{\prime}}}
$$

where $\sigma^{\prime}, r_{j}^{\prime} \in \mathbf{N}$, the $\tilde{p}^{j}(t, \tau, \xi ; \varepsilon)(\in \Sigma[\tau])$ are irreducible in $\Sigma[\tau]$ and $\tilde{p}^{j}(t, \tau, \xi ; \varepsilon)$ and $\tilde{p}^{k}(t, \tau, \xi ; \varepsilon)$ are mutually prime if $j \neq k$. Put

$$
\tilde{q}(t, \tau, \xi ; \varepsilon)=\prod_{j=1}^{\sigma^{\prime}} \tilde{p}^{j}(t, \tau, \xi ; \varepsilon),
$$

and let $\tilde{D}(t, \xi ; \varepsilon)$ be the discriminant of $\tilde{q}(t, \tau, \xi ; \varepsilon)=0$ in $\tau$. Then we can write

$$
\tilde{D}(t, \xi ; \varepsilon)=\tilde{d}_{0}(t, \xi ; \varepsilon) / \tilde{d}_{1}(t, \xi ; \varepsilon),
$$

where $\tilde{d}_{k}(t, \xi ; \varepsilon) \in \mathscr{A}[\xi, \varepsilon]$ and $\tilde{d}_{k}(t, \xi ; \varepsilon) \neq 0$ in $\mathscr{A}[\xi, \varepsilon](k=0,1)$. Put

$$
\tilde{\mathscr{N}}_{1}=\tilde{\mathscr{N}}_{0} \cup\left\{(\xi, \varepsilon) \in\left(\mathbf{R}^{n} \backslash\{0\}\right) \times \mathbf{R} ; \tilde{d}_{0}(t, \xi ; \varepsilon) \tilde{d}_{1}(t, \xi ; \varepsilon) \equiv 0 \text { in } t\right\} .
$$

Then we have, similarly, $\mu_{n+1}\left(\tilde{\mathscr{N}}_{1}\right)=0$. Define

$$
\mathscr{N}_{1}^{0}=\left\{\varepsilon \in \mathbf{R} ; \mu_{n}\left(\left\{\xi \in \mathbf{R}^{n} \backslash\{0\} ;(\xi, \varepsilon) \in \tilde{\mathscr{N}}_{1}\right\}\right)>0\right\} .
$$

Then we have $\mu_{1}\left(\mathscr{N}_{1}^{0}\right)=0$. For $\varepsilon \in \mathbf{R} \backslash \mathscr{N}_{1}^{0}$ we define

$$
\mathscr{N}_{1}(\varepsilon)=\left\{\xi \in \mathbf{R}^{n} \backslash\{0\} ;(\xi, \varepsilon) \in \tilde{\mathscr{N}}_{1}\right\} .
$$

By definition we have

$$
\begin{aligned}
& \mu_{n}\left(\mathscr{N}_{1}(\varepsilon)\right)=0 \text { for } \varepsilon \in \mathbf{R} \backslash \mathscr{N}_{1}^{0} \\
& \tilde{d}_{0}(t, \xi ; \varepsilon) \tilde{d}_{1}(t, \xi ; \varepsilon) \not \equiv 0 \text { in } t \text { for } \varepsilon \in \mathbf{R} \backslash \mathscr{N}_{1}^{0} \text { and } \xi \in S^{n-1} \backslash \mathscr{N}_{1}(\varepsilon) .
\end{aligned}
$$

We may assume that the $\tilde{d}_{k}(t, \xi ; \varepsilon)$ are homogeneous in $\xi$. So $\mathscr{N}_{1}(\varepsilon)$ is a cone. For $\varepsilon \in \mathbf{R} \backslash \mathscr{N}_{1}^{0}$ and $\xi \in S^{n-1} \backslash \mathscr{N}_{1}(\varepsilon)$ the roots of $\tilde{q}(t, \tau, \xi ; \varepsilon)=0$ in $\tau$ are simple 
if $t \in[0, \infty)$ and $\tilde{d}_{0}(t, \xi ; \varepsilon) \tilde{d}_{1}(t, \xi ; \varepsilon) \neq 0$. Therefore, the multiplicities of the roots $\partial_{t} \lambda_{j}(t, \xi ; \varepsilon)$ of $\tilde{p}(t, \tau, \xi ; \varepsilon)=0$ in $\tau(1 \leq j \leq \hat{m})$ are constant for $\varepsilon \in \mathbf{R} \backslash \mathscr{N}_{1}^{0}, \xi \in$ $S^{n-1} \backslash \mathscr{N}_{1}(\varepsilon)$ and $t \in[0, \infty)$ with $\tilde{d}_{0}(t, \xi ; \varepsilon) \tilde{d}_{1}(t, \xi ; \varepsilon) \neq 0$. Since $\tilde{d}_{0}(t, \xi ; \varepsilon) \tilde{d}_{1}(t, \xi ; \varepsilon) \in$ $\mathscr{A}[\xi, \varepsilon]$, it follows from Lemma 2.1 that for any $T>0$ there is $N_{T, 1} \in \mathbf{Z}_{+}$such that

$$
\begin{aligned}
& \#\left\{t \in[0, T] ; \partial_{t}\left(\lambda_{j}(t, \xi ; \varepsilon)-\lambda_{k}(t, \xi ; \varepsilon)\right)=0\right\} \\
& \left(\leq \#\left\{t \in[0, T] ; \tilde{d}_{0}(t, \xi ; \varepsilon) \tilde{d}_{1}(t, \xi ; \varepsilon)=0\right\}\right) \leq N_{T, 1}
\end{aligned}
$$

if $\varepsilon \in \mathbf{R} \backslash \mathscr{N}_{1}^{0}, \xi \in S^{n-1} \backslash \mathscr{N}_{1}(\varepsilon), 1 \leq j<k \leq m$ and $\partial_{t}\left(\lambda_{j}(t, \xi ; \varepsilon)-\lambda_{k}(t, \xi ; \varepsilon)\right) \not \equiv 0$ in $t$. This proves the second part of the assertions of the lemma in the case where $v=1$. Repeating the above arguments we can prove the lemma for $v=2,3, \ldots$, inductively.

Lemma 2.4. Let $T>0, \Gamma$ be a cone (with its vertex at 0 ) in $\mathbf{R}^{n} \backslash\{0\}$, and let $a(t, \xi)$ be a function defined for $(t, \xi) \in[0, T] \times \Gamma$ satisfying the following:

(i) $a(t, \xi)$ is continuously differentiable in $t \in[0, T]$ and positively homogeneous of degree 1 in $\xi$.

(ii) $\#\left\{t \in[0, T] ; \partial_{t} a(t, \xi)=0\right\} \leq N$ if $\xi \in \Gamma$ and $\partial_{t} a(t, \xi) \not \equiv 0$ in $t$.

(iii) $|a(t, \xi)| \leq C_{0}|\xi|$ for $t \in[0, T]$ and $\xi \in \Gamma$.

Here $N \in \mathbf{Z}_{+}$and $C_{0} \geq 0$. Then there is a positive constant $C\left(N, C_{0}\right)$, which depends only on $N$ and $C_{0}$, such that

$$
\begin{aligned}
& \int_{0}^{T}\left|\partial_{t} a(t, \xi)\right| /|\xi| d t \leq C\left(N, C_{0}\right) \\
& \int_{0}^{T}\left|\partial_{t} a(t, \xi)\right| /(|a(t, \xi)|+1) d t \leq C\left(N, C_{0}\right)(\log \langle\xi\rangle+1)
\end{aligned}
$$

for $\xi \in \Gamma$.

Proof. Fix $\xi \in \Gamma$. We may assume that $\partial_{t} a(t, \xi) \not \equiv 0$ in $t$. Noting that $\#\{t \in[0, T] ; a(t, \xi)=0\} \leq N+1$, we write

$$
\#\left\{t \in[0, T] ; a(t, \xi) \partial_{t} a(t, \xi)=0\right\}=\left\{t_{1}, t_{2}, \ldots, t_{N(\xi)}\right\},
$$

where $N(\xi) \in \mathbf{Z}_{+}$and $0 \leq t_{1}<t_{2}<\cdots<t_{N(\xi)} \leq T$. It is obvious that $N(\xi) \leq$ $2 N+1$. In each sub-interval $\left[t_{j-1}, t_{j}\right](1 \leq j \leq N(\xi)+1)$ we have " $a(t, \xi) \geq 0$ or 
$a(t, \xi) \leq 0$ " and " $\partial_{t} a(t, \xi) \geq 0$ or $\partial_{t} a(t, \xi) \leq 0$ ", where $t_{0}=0$ and $t_{N(\xi)+1}=T$. Then we have

$$
\int_{t_{j-1}}^{t_{j}}\left|\partial_{t} a(t, \xi)\right| /|\xi| d t=\left|a\left(t_{j}, \xi /|\xi|\right)-a\left(t_{j-1}, \xi /|\xi|\right)\right| \leq 2 C_{0} .
$$

Moreover, we have

$$
\int_{t_{j-1}}^{t_{j}}\left|\partial_{t} a(t, \xi)\right| /(|a(t, \xi)|+1) d t \leq \int_{t_{j-1}}^{t_{j}}\left|\partial_{t} a(t, \xi /|\xi|)\right| d t \leq 2 C_{0} \quad \text { if }|\xi| \leq 1 .
$$

If $|\xi| \geq 1$, then

$$
\begin{aligned}
& \int_{t_{j-1}}^{t_{j}}\left|\partial_{t} a(t, \xi)\right| /(|a(t, \xi)|+1) d t \\
& \quad=\left|\log \left(\left|a\left(t_{j}, \xi /|\xi|\right)\right|+|\xi|^{-1}\right)-\log \left(\left|a\left(t_{j-1}, \xi /|\xi|\right)\right|+|\xi|^{-1}\right)\right| \\
& \quad \leq \log \left(C_{0}+1\right)+\log |\xi| .
\end{aligned}
$$

Therefore, we have

$$
\begin{aligned}
& \int_{0}^{T}\left|\partial_{t} a(t, \xi)\right| /|\xi| d t \leq 2 C_{0}(2 N+1), \\
& \int_{0}^{T}\left|\partial_{t} a(t, \xi)\right| /(|a(t, \xi)|+1) d t \leq \begin{cases}2 C_{0}(2 N+1) & \text { if }|\xi| \leq 1, \\
(2 N+1)\left(\log \left(C_{0}+1\right)+\log \langle\xi\rangle\right) & \text { if }|\xi| \geq 1,\end{cases}
\end{aligned}
$$

which proves the lemma.

Put

$$
p_{\varepsilon}^{(j)}(t, \tau, \xi)=\partial_{\tau}^{j} p_{\varepsilon}(t, \tau, \xi)\left(=\left(1-\varepsilon|\xi|^{2} \partial_{\tau}^{2}\right) \partial_{\tau}^{j} p(t, \tau, \xi)\right)
$$

$(1 \leq j \leq m)$ and write

$$
p_{\varepsilon}^{(j)}(t, \tau, \xi)=(m ! /(m-j) !) \prod_{k=1}^{m-j}\left(\tau-\lambda_{k}^{(j)}(t, \xi ; \varepsilon)\right) \quad(1 \leq j \leq m-1) .
$$

Here we enumerate so that the $\lambda_{k}^{(j)}(t, \xi ; \varepsilon)$ are real analytic in $t \in[0, \infty)$. Recall that

$$
\left|p_{\varepsilon}^{(j)}(t, \tau-i \gamma, \xi)\right|^{2}=\sum_{k=0}^{m-j} \gamma^{2 k} h_{m-j-k}\left(t, \tau, \xi ; p_{\varepsilon}^{(j)}\right)
$$

for $t \in[0, \infty),(\tau, \xi) \in \mathbf{R}^{n+1}, \varepsilon \in \mathbf{R}$ and $\gamma \in \mathbf{R}$. Then it follows from Lemma 2.1 of [11] that 


$$
\begin{aligned}
(m-r) !(k-r) ! /(m ! k !) & \leq h_{m-k}\left(t, \tau, \xi ; p_{\varepsilon}\right) / h_{m-k}\left(t, \tau, \xi ; p_{\varepsilon}^{(r)}\right) \\
& \leq(k-1-r) !(k-r) ! /((k-1) ! k !)
\end{aligned}
$$

for $0 \leq r<k \leq m$ (see, also, (2.3)). We put

$$
\begin{aligned}
& p_{I}(t, \tau, \xi ; \varepsilon)=\prod_{j \in\{1,2, \ldots, m\} \backslash I}\left(\tau-\lambda_{j}(t, \xi ; \varepsilon)\right) \quad \text { for } I \subset\{1,2, \ldots, m\}, \\
& p_{j}(t, \tau, \xi ; \varepsilon)=p_{\{j\}}(t, \tau, \xi ; \varepsilon), \\
& p_{j, k}(t, \tau, \xi ; \varepsilon)=p_{\{j, k\}}(t, \tau, \xi ; \varepsilon) \quad(j \neq k), \cdots, \\
& p_{j}^{(k)}(t, \tau, \xi ; \varepsilon)=(m ! /(m-k) !) \prod_{l \neq j}\left(\tau-\lambda_{l}^{(k)}(t, \xi ; \varepsilon)\right) .
\end{aligned}
$$

Note that

$$
h_{m-j}\left(t, \tau, \xi ; p_{\varepsilon}\right)=\sum_{\substack{I \subset\{1,2, \ldots, m\} \\ \# I=j}} p_{I}(t, \tau, \xi ; \varepsilon)^{2} .
$$

Lemma 2.5. Let $r \in \mathbf{N}, \tau_{j} \in \mathbf{R}(1 \leq j \leq r)$ and $a_{k} \in \mathbf{C}(0 \leq k \leq r-1)$, and put $p(\tau)=a_{0} \tau^{r-1}+a_{1} \tau^{r-2}+\cdots+a_{r-1}$. If

$$
|p(\tau)| \leq A \sum_{j=1}^{r} \prod_{1 \leq k \leq r, k \neq j}\left|\tau-\tau_{k}\right| \quad \text { for any } \tau \in \mathbf{R},
$$

then there are $b_{j}(1 \leq j \leq r)$ such that $\left|b_{j}\right| \leq A(1 \leq j \leq r)$ and

$$
p(\tau)=\sum_{j=1}^{r} b_{j} \prod_{1 \leq k \leq r, k \neq j}\left(\tau-\tau_{k}\right) .
$$

Proof. Write

$$
\prod_{k=1}^{r}\left(\tau-\tau_{k}\right)=\prod_{j=1}^{r_{0}}\left(\tau-\lambda_{j}\right)^{m_{j}}
$$

where $r_{0} \in \mathbf{N}, m_{j} \in \mathbf{N}, \lambda_{j} \neq \lambda_{j^{\prime}}\left(j \neq j^{\prime}\right)$ and $\left\{\tau_{1}, \ldots, \tau_{r}\right\}=\left\{\lambda_{1}, \ldots, \lambda_{r_{0}}\right\}$. Then we have $m_{1}+\cdots+m_{r_{0}}=r$ and

$$
|p(\tau)| \leq A \prod_{j=1}^{r_{0}}\left|\tau-\lambda_{j}\right|^{m_{j}-1} \sum_{j=1}^{r_{0}} m_{j} \prod_{k \neq j}\left|\tau-\lambda_{k}\right| .
$$


Therefore, there are a polynomial $\tilde{p}(\tau)$ of $\tau$ such that $\operatorname{deg} \tilde{p}=r_{0}-1$ and

$$
p(\tau)=\tilde{p}(\tau) \prod_{j=1}^{r_{0}}\left(\tau-\lambda_{j}\right)^{m_{j}-1} .
$$

Since

$$
|\tilde{p}(\tau)| \leq A \sum_{j=1}^{r_{0}} m_{j} \prod_{k \neq j}\left|\tau-\lambda_{k}\right|,
$$

Lagrange's interpolation formula gives

$$
\tilde{p}(\tau)=\sum_{j=1}^{r_{0}}\left\{\tilde{p}\left(\lambda_{j}\right) / \prod_{k \neq j}\left(\lambda_{j}-\lambda_{k}\right)\right\} \prod_{k \neq j}\left(\tau-\lambda_{k}\right) .
$$

Thus, putting

$$
b_{j}=\tilde{p}\left(\lambda_{l}\right) /\left(m_{l} \prod_{k \neq l}\left(\lambda_{l}-\lambda_{k}\right)\right) \quad \text { if } \tau_{j}=\lambda_{l},
$$

we have (2.12). (2.13) with $\tau=\lambda_{l}$ and (2.14) give $\left|b_{j}\right| \leq A(1 \leq j \leq r)$.

Lemma 2.6. (i) The condition (D-L) is equivalent to the following condition $(\mathrm{D}-\mathrm{L})^{\prime}$ :

(D-L)' There are $b_{1, j}(t, \xi ; \varepsilon)(1 \leq j \leq m)$ defined for $\xi \in \mathbf{R}^{n} \backslash\{0\}, t \in[0, \infty) \backslash$ $\mathscr{R}(\xi /|\xi|)$ and $\varepsilon \in \mathbf{R}$ such that the $b_{1, j}(t, \xi ; \varepsilon)$ are positively homogeneous in $\xi$ of degree 0 ,

$$
\begin{aligned}
& \text { sub } \sigma(P)(t, \tau, \xi)=\sum_{j=1}^{m} b_{1, j}(t, \xi ; \varepsilon) p_{j}(t, \tau, \xi ; \varepsilon) \\
& \text { for } \xi \in S^{n-1}, t \in[0, \infty) \backslash \mathscr{R}(\xi) \text { and } \varepsilon \in \mathbf{R},
\end{aligned}
$$

and for any $T>0$ there is $C>0$ satisfying

$$
\begin{aligned}
& \min \left\{\min _{s \in \mathscr{R}(\xi)}|t-s|, 1\right\}\left|b_{1, j}(t, \xi ; \varepsilon)\right| \leq C \\
& \quad \text { for } 1 \leq j \leq m, \xi \in S^{n-1}, t \in[0, \infty) \backslash \mathscr{R}(\xi) \text { and } \varepsilon \in \mathbf{R} .
\end{aligned}
$$

(ii) Assume that $m=3$. Then the condition (T-L) is equivalent to the following condition $(\mathrm{T}-\mathrm{L})^{\prime}$ : 
(T-L)' The condition (D-L)' is satisfied, and there are $b_{2, j}(t, \xi ; \varepsilon)(j=1,2)$ defined for $\xi \in \mathbf{R}^{n} \backslash\{0\}, t \in[0, \infty) \backslash \mathscr{R}(\xi /|\xi|)$ and $\varepsilon \in \mathbf{R}$ such that the $b_{2, j}(t, \xi ; \varepsilon)$ are positively homogeneous in $\xi$ of degree 0 ,

$$
\begin{array}{r}
s u b^{2} \sigma(P)(t, \tau, \xi)=\sum_{j=1}^{2} b_{2, j}(t, \xi ; \varepsilon) p_{j}^{(1)}(t, \tau, \xi ; \varepsilon) \\
\text { for } \xi \in S^{n-1}, t \in[0, \infty) \backslash \mathscr{R}(\xi) \text { and } \varepsilon \in \mathbf{R},
\end{array}
$$

and for any $T>0$ there is $C>0$ satisfying

$$
\begin{aligned}
& \min \left\{\min _{s \in \mathscr{R}(\xi)}|t-s|^{2}, 1\right\}\left|b_{2, j}(t, \xi ; \varepsilon)\right| \leq C \\
& \quad \text { for } j=1,2, \xi \in S^{n-1}, t \in[0, \infty) \backslash \mathscr{R}(\xi) \text { and } \varepsilon \in \mathbf{R} .
\end{aligned}
$$

Proof. It is obvious that (D-L) with $C$ replaced by $\sqrt{m} C$ is valid if $(\mathrm{D}-\mathrm{L})^{\prime}$ with $\varepsilon=0$ is valid, since we have, by (2.11),

$$
\sum_{j=1}^{m}\left|p_{j}(t, \tau, \xi ; 0)\right| \leq \sqrt{m} h_{m-1}(t, \tau, \xi ; p)^{1 / 2} .
$$

Similarly, from (2.10) and (2.11) it follows that (1.2) with $C$ replaced by $2 \sqrt{3} C$ in (T-L) is valid if (T-L)' with $\varepsilon=0$ is valid. The converses in (i) and (ii) easily follow from Lemma 2.5 .

COROLlaRY 2.7. Let $\delta>0$ and $\tau_{j} \in \mathbf{R} \quad(1 \leq j \leq r)$ satisfy $\left|\tau_{j}-\tau_{k}\right| \geq \delta$ $(1 \leq j<k \leq r)$. Then there are $b_{l, j} \in \mathbf{C}(0 \leq l \leq r-1,1 \leq j \leq r)$ such that

$$
\begin{aligned}
& \left|b_{l, j}\right| \leq \delta^{-r+1}\left|\tau_{j}\right|^{l} \\
& \tau^{l}=\sum_{j=1}^{r} b_{l, j} \prod_{k \neq j}\left(\tau-\tau_{k}\right) .
\end{aligned}
$$

Proof. Take $b_{l, j}=\tau_{j}^{l} / \prod_{k \neq j}\left(\tau_{j}-\tau_{k}\right)$. Then (2.16) and (2.17) are satisfied.

Define

$$
\mathscr{P}_{j}(t, \tau, \xi ; \varepsilon)=p_{j}(t, \tau, \xi ; \varepsilon)-(i / 2) \partial_{t} \partial_{\tau} p_{j}(t, \tau, \xi ; \varepsilon) \quad(1 \leq j \leq m)
$$


Then a simple calculation yields

$$
\begin{aligned}
(\tau- & \left.\lambda_{j}(t, \xi ; \varepsilon)\right) \circ \mathscr{P}_{j}(t, \tau, \xi ; \varepsilon) \\
= & p_{\varepsilon}(t, \tau, \xi)-(i / 2) \partial_{t} \partial_{\tau} p_{\varepsilon}(t, \tau, \xi) \\
& \quad-(i / 2) \sum_{k \neq j} \partial_{t}\left(\lambda_{j}(t, \xi ; \varepsilon)-\lambda_{k}(t, \xi ; \varepsilon)\right) \cdot p_{j, k}(t, \tau, \xi ; \varepsilon) \\
& \quad-\partial_{t}^{2} \partial_{\tau} p_{j}(t, \tau, \xi ; \varepsilon) / 2 \text { for } 1 \leq j \leq m,
\end{aligned}
$$

where $a(t, \tau, \xi) \circ b(t, \tau, \xi)$ denotes the symbol of $a\left(t, D_{t}, \xi\right) b\left(t, D_{t}, \xi\right)$. Indeed, we have

$$
\begin{aligned}
& \left(\tau-\lambda_{j}(t, \xi ; \varepsilon)\right) \circ p_{j}(t, \tau, \xi ; \varepsilon)=p_{\varepsilon}(t, \tau, \xi)-i \partial_{t} p_{j}(t, \tau, \xi ; \varepsilon), \\
& \left(\tau-\lambda_{j}(t, \xi ; \varepsilon)\right) \circ \partial_{t} \partial_{\tau} p_{j}(t, \tau, \xi ; \varepsilon)=\partial_{t} \partial_{\tau} p_{\varepsilon}(t, \tau, \xi)-\partial_{t} p_{j}(t, \tau, \xi ; \varepsilon) \\
& +\partial_{t} \lambda_{j}(t, \xi ; \varepsilon) \cdot \partial_{\tau} p_{j}(t, \tau, \xi ; \varepsilon)-i \partial_{t}^{2} \partial_{\tau} p_{j}(t, \tau, \xi ; \varepsilon) .
\end{aligned}
$$

From (2.19) we have

$$
\begin{aligned}
\left(\tau-\lambda_{j}(t, \xi ; \varepsilon)\right) \circ \mathscr{P}_{j}(t, \tau, \xi ; \varepsilon) \\
=p_{\varepsilon}(t, \tau, \xi)-(i / 2) \partial_{t} \partial_{\tau} p_{\varepsilon}(t, \tau, \xi)-(2 m)^{-1} \partial_{t}^{2} \partial_{\tau}^{2} p_{\varepsilon}(t, \tau, \xi) \\
\quad-(i / 2) \sum_{k \neq j} \partial_{t}\left(\lambda_{j}(t, \xi ; \varepsilon)-\lambda_{k}(t, \xi ; \varepsilon)\right) \cdot p_{j, k}(t, \tau, \xi ; \varepsilon) \\
\quad-(2 m)^{-1} \partial_{t}^{2}\left\{\sum_{k \neq j} \sum_{l \neq j, k}\left(\lambda_{j}(t, \xi ; \varepsilon)-\lambda_{k}(t, \xi ; \varepsilon)\right) p_{j, k, l}(t, \tau, \xi ; \varepsilon)\right\}
\end{aligned}
$$

for $1 \leq j \leq m$. Note that $p_{j, k, l}(t, \tau, \xi ; \varepsilon)=1$ if $m=3$. We have also

$$
\left(\tau-\lambda_{j}^{(r)}(t, \xi ; \varepsilon)\right) \circ p_{j}^{(r)}(t, \tau, \xi ; \varepsilon)=p_{\varepsilon}^{(r)}(t, \tau, \xi)-i \partial_{t} p_{j}^{(r)}(t, \tau, \xi ; \varepsilon)
$$

for $1 \leq r \leq m-1$ and $1 \leq j \leq m-r$. In particular, we have

$$
\begin{aligned}
(\tau- & \left.\lambda_{j}^{(1)}(t, \xi ; \varepsilon)\right) \circ p_{j}^{(1)}(t, \tau, \xi ; \varepsilon) \\
& =\sum_{l=1}^{3} \mathscr{P}_{l}(t, \tau, \xi ; \varepsilon)+(i / 2) \partial_{t}\left(\lambda_{j}^{(1)}(t, \xi ; \varepsilon)-\lambda_{k}^{(1)}(t, \xi ; \varepsilon)\right)
\end{aligned}
$$


if $m=3$ and $\{j, k\}=\{1,2\}$. Indeed, we have

$$
\begin{aligned}
& \partial_{t} \partial_{\tau}^{2} p_{\varepsilon}(t, \tau, \xi)=\partial_{t} \partial_{\tau} p_{\varepsilon}^{(1)}(t, \tau, \xi)=\sum_{k=1}^{2} \partial_{t} p_{k}^{(1)}(t, \tau, \xi ; \varepsilon), \\
& p_{\varepsilon}^{(1)}(t, \tau, \xi ; \varepsilon)-(i / 2) \partial_{t} \partial_{\tau}^{2} p_{\varepsilon}(t, \tau, \xi)=\sum_{l=1}^{3} \mathscr{P}_{l}(t, \tau, \xi ; \varepsilon) .
\end{aligned}
$$

\section{Proof of Theorem $\mathbf{1 . 2}$}

In this section we assume that the hypotheses of Theorem 1.2 are fulfilled and we shall prove Theorem 1.2. Let $\left\{\varepsilon_{j}\right\}_{j=1,2, \ldots}$ be a sequence satisfying $\varepsilon_{j} \in(0,1] \backslash \mathscr{N}_{2}^{0}$ and $\varepsilon_{j} \downarrow 0$ as $j \rightarrow \infty$, where $\mathscr{N}_{2}^{0}$ is as in Lemma 2.3. Put

$$
\begin{aligned}
& E_{0}=\left\{\varepsilon_{j} ; j=1,2, \ldots\right\} \cup\{0\}, \\
& \mathscr{N}=\bigcup_{j=1}^{\infty} \mathscr{N}_{2}\left(\varepsilon_{j}\right) \cup \mathscr{N}_{2}(0) \cup\{0\}\left(\subset \mathbf{R}^{n}\right),
\end{aligned}
$$

where $\mathscr{N}_{2}(\varepsilon)$ is as in Lemma 2.3. Note that $\mu_{n}(\mathscr{N})=0$. We define

$$
\begin{aligned}
P_{\varepsilon}(t, \tau, \xi)= & P(t, \tau, \xi)+p_{\varepsilon}(t, \tau, \xi)-p(t, \tau, \xi) \\
& -(i / 2) \partial_{t} \partial_{\tau}\left(p_{\varepsilon}(t, \tau, \xi)-p(t, \tau, \xi)\right)
\end{aligned}
$$

Note that

$$
\begin{aligned}
& \operatorname{sub} \sigma\left(P_{\varepsilon}\right)(t, \tau, \xi)=\operatorname{sub} \sigma(P)(t, \tau, \xi) \\
& P_{\varepsilon}(t, \tau, \xi)=P(t, \tau, \xi)+p_{\varepsilon}(t, \tau, \xi)-p(t, \tau, \xi) \quad \text { if } m=3
\end{aligned}
$$

Consider the Cauchy problem

$(\mathrm{CP})_{\varepsilon} \quad\left\{\begin{array}{l}P_{\varepsilon}\left(t, D_{t}, D_{x}\right) u_{\varepsilon}(t, x)=f(t, x) \quad \text { in }[0, \infty) \times \mathbf{R}^{n}, \\ \left.D_{t}^{j} u_{\varepsilon}(t, x)\right|_{t=0}=u_{j}(x) \text { in } \mathbf{R}^{n}(0 \leq j \leq m-1)\end{array}\right.$

for $\varepsilon \in E_{0}$, where $f \in C^{\infty}\left([0, \infty) ; H^{\infty}\left(\mathbf{R}_{x}^{n}\right)\right)$ and $u_{j} \in H^{\infty}\left(\mathbf{R}^{n}\right) \quad(0 \leq j \leq m-1)$. Here $H^{s}\left(\mathbf{R}^{n}\right)$ denotes the Sobolev space over $\mathbf{R}^{n}$ of order $s$ and $H^{\infty}\left(\mathbf{R}^{n}\right)=$ $\bigcap_{s \in \mathbf{R}} H^{s}\left(\mathbf{R}^{n}\right)$. By partial Fourier transformation in $x$, the Cauchy problem $(\mathrm{CP})_{\varepsilon}$ is reduced to the Cauchy problem for an ordinary differential operator with parameters $\xi$ : 


$$
\left\{\begin{array}{l}
P_{\varepsilon}\left(t, D_{t}, \xi\right) v_{\varepsilon}(t, \xi)=\hat{f}(t, \xi) \quad \text { for }(t, \xi) \in[0, \infty) \times \mathbf{R}^{n} \\
\left.D_{t}^{j} v_{\varepsilon}(t, \xi)\right|_{t=0}=\hat{u}_{j}(\xi) \text { for } \xi \in \mathbf{R}^{n}(0 \leq j \leq m-1)
\end{array}\right.
$$

for $\varepsilon \in E_{0}$, where $\hat{f}(t, \xi)$ and $\hat{u}_{j}(\xi)(0 \leq j \leq m-1)$ denotes the partial Fourier transforms of $f(t, x)$ and $u_{j}(x)$ with respect to $x$, respectively, for example, $\hat{f}(t, \xi)=\int_{\mathbf{R}^{n}} e^{-i x \cdot \xi} f(t, x) d x$. We note that the Cauchy problem (3.1) has a unique solution $v_{\varepsilon}(t, \xi) \in C^{\infty}\left([0, \infty) ; C^{\infty}\left(\mathbf{R}_{\xi}^{n}\right)\right)$. If it can be shown that $v_{\varepsilon}(t, \xi) \in$ $C^{m}\left([0, \infty) ; \mathscr{S}^{\prime}\left(\mathbf{R}_{\xi}^{n}\right)\right)$, then $u_{\varepsilon}(t, x)=\mathscr{F}_{\xi}^{-1}\left[v_{\varepsilon}(t, \xi)\right](x) \quad\left(\in C^{m}\left([0, \infty) ; \mathscr{S}^{\prime}\left(\mathbf{R}_{x}^{n}\right)\right)\right.$ is a unique solution to the Cauchy problem $(\mathrm{CP})_{\varepsilon}$, where $\mathscr{F}_{\xi}^{-1}[v(t, \xi)](x)$ denotes the inverse partial Fourier transform of $v(t, \xi)$ in $\xi$. We fix $T>0$. Define

$$
\begin{aligned}
& W_{0}(t, \xi)=\sum_{s \in \mathscr{R}(\xi / \xi \mid) \cap[0, T+1]}\langle\xi\rangle^{1 / 2} / \sqrt{(t-s)^{2}\langle\xi\rangle+1}+1, \\
& W_{1}(t, \xi ; \varepsilon)=\sum_{1 \leq j<k \leq m}\left|\partial_{t}\left(\lambda_{j}(t, \xi ; \varepsilon)-\lambda_{k}(t, \xi ; \varepsilon)\right)\right| /\left(\left|\lambda_{j}(t, \xi ; \varepsilon)-\lambda_{k}(t, \xi ; \varepsilon)\right|+1\right) \\
& +\sum_{j=1}^{m}\left|\partial_{t}^{2} \lambda_{j}(t, \xi ; \varepsilon)\right| /|\xi|, \\
& \Lambda(t, \xi ; \varepsilon)=\int_{0}^{t}\left(W_{0}(s, \xi)+W_{1}(s, \xi ; \varepsilon)\right) d s
\end{aligned}
$$

for $(t, \xi, \varepsilon) \in[0, T] \times\left(\mathbf{R}^{n} \backslash \mathscr{N}\right) \times E_{0}$, where $\langle\xi\rangle=\left(1+|\xi|^{2}\right)^{1 / 2}$. Note that

$$
\begin{aligned}
& \partial_{t} \log \left(\sqrt{(t-s)^{2}\langle\xi\rangle+1}+(t-s)\langle\xi\rangle^{1 / 2}\right)=\langle\xi\rangle^{1 / 2} / \sqrt{(t-s)^{2}\langle\xi\rangle+1} \\
& \left|\partial_{t} W_{0}(t, \xi)\right| \leq \sum_{s \in \mathscr{R}(\xi / \mid \xi) \cap[0, T+1]}\langle\xi\rangle /\left((t-s)^{2}\langle\xi\rangle+1\right) \leq W_{0}(t, \xi)^{2} .
\end{aligned}
$$

From (3.2) and Lemmas 2.3 and 2.4 it follows that there is $C_{T}>0$ satisfying

$$
0 \leq \Lambda(t, \xi ; \varepsilon) \leq C_{T}(\log \langle\xi\rangle+1) \quad \text { for } t \in[0, T], \xi \in \mathbf{R}^{n} \backslash \mathscr{N} \text { and } \varepsilon \in E_{0}
$$

Here we have used the fact that, with some $C_{T}^{\prime}>0$,

$$
\left|\partial_{t} \lambda_{j}(t, \xi ; \varepsilon)\right| \leq C_{T}^{\prime}|\xi| \text { for }(t, \xi, \varepsilon) \in[0, T] \times \mathbf{R}^{n} \times[-1,1]
$$

(see, e.g., Theorem 1 of [14]). For $(t, \xi, \varepsilon) \in[0, T] \times\left(\mathbf{R}^{n} \backslash \mathscr{N}\right) \times E_{0}$ and $A \geq 1$ we define 


$$
\begin{aligned}
\mathscr{E}(t, \xi ; \varepsilon ; A)= & \sum_{j=1}^{m} e^{-A \Lambda(t, \xi ; \varepsilon)}\left|\mathscr{P}_{j}\left(t, D_{t}, \xi ; \varepsilon\right) v_{\varepsilon}(t, \xi)\right|^{2} \\
& +\sum_{k=1}^{m-1} \sum_{j=1}^{m-k} W_{0}(t, \xi)^{2} e^{-A \Lambda(t, \xi ; \varepsilon)}\left|p_{j}^{(k)}\left(t, D_{t}, \xi ; \varepsilon\right) v_{\varepsilon}(t, \xi)\right|^{2}
\end{aligned}
$$

Let $(t, \xi) \in[0, T] \times\left(\mathbf{R}^{n} \backslash \mathscr{N}\right)$ and $\varepsilon \in E_{0}$. It is obvious that

$$
\begin{gathered}
D_{t} \mathscr{E}(t, \xi ; \varepsilon ; A)=i \sum_{j=1}^{m}\left[A \Lambda_{t} e^{-A \Lambda}\left|\mathscr{P}_{j} v_{\varepsilon}\right|^{2}+2 \operatorname{Im}\left\{e^{-A \Lambda}\left(D_{t} \mathscr{P}_{j} v_{\varepsilon}\right) \cdot\left(\overline{\mathscr{P}_{j} v_{\varepsilon}}\right)\right\}\right] \\
+i \sum_{k=1}^{m-1} \sum_{j=1}^{m-k}\left[A W_{0}^{2} \Lambda_{t} e^{-A \Lambda}\left|p_{j}^{(k)} v_{\varepsilon}\right|^{2}-2 W_{0} W_{0 t} e^{-A \Lambda}\left|p_{j}^{(k)} v_{\varepsilon}\right|^{2}\right. \\
\left.+2 \operatorname{Im}\left\{W_{0}^{2} e^{-A \Lambda}\left(D_{t} p_{j}^{(k)} v_{\varepsilon}\right) \cdot\left(\overline{p_{j}^{(k)} v_{\varepsilon}}\right)\right\}\right] .
\end{gathered}
$$

Here we write $\Lambda=\Lambda(t, \xi ; \varepsilon), \Lambda_{t}=\partial_{t} \Lambda(t, \xi ; \varepsilon), \mathscr{P}_{j}=\mathscr{P}_{j}(t, \tau, \xi ; \varepsilon), v_{\varepsilon}=v_{\varepsilon}(t, \xi), W_{0}=$ $W_{0}(t, \xi), W_{0 t}=\partial_{t} W_{0}(t, \xi)$ and so forth. Since the $\lambda_{j}(t, \xi ; \varepsilon)$ are real-valued, from (2.19) we have

$$
\begin{aligned}
\operatorname{Im}\left\{e^{-A \Lambda}\left(D_{t} \mathscr{P}_{j} v_{\varepsilon}\right) \cdot\left(\overline{\mathscr{P}_{j} v_{\varepsilon}}\right)\right\} \\
=\operatorname{Im}\left\{e^{-A \Lambda}\left(\left(D_{t}-\lambda_{j}(t, \xi ; \varepsilon)\right) \mathscr{P}_{j} v_{\varepsilon}\right) \cdot\left(\overline{\mathscr{P}_{j} v_{\varepsilon}}\right)\right\} \\
=\operatorname{Im}\left\{e^{-A \Lambda}\left(\left(p_{\varepsilon}-(i / 2)\left(\partial_{t} \partial_{\tau} p_{\varepsilon}\right)\left(t, D_{t}, \xi\right)\right) v_{\varepsilon}\right) \cdot\left(\overline{\mathscr{P}_{j} v_{\varepsilon}}\right)\right\} \\
\quad-\operatorname{Re}\left\{e^{-A \Lambda} \sum_{k \neq j}\left(\lambda_{j t}-\lambda_{k t}\right)\left(p_{j, k} v_{\varepsilon}\right) \cdot\left(\overline{\mathscr{P}_{j} v_{\varepsilon}}\right)\right\} / 2 \\
\quad-\operatorname{Im}\left\{e^{-A \Lambda}\left(\left(\partial_{t}^{2} \partial_{\tau} p_{j}\right)\left(t, D_{t}, \xi ; \varepsilon\right) v_{\varepsilon}\right) \cdot\left(\overline{\mathscr{P}_{j} v_{\varepsilon}}\right)\right\} / 2 \quad(1 \leq j \leq m) .
\end{aligned}
$$

Similarly, (2.21) gives

$$
\begin{aligned}
\operatorname{Im}\{ & \left.\left.W_{0}^{2} e^{-A \Lambda}\left(D_{t} p_{j}^{(k)} v_{\varepsilon}\right) \cdot \overline{\left(p_{j}^{(k)} v_{\varepsilon}\right.}\right)\right\} \\
= & \operatorname{Im}\left\{W_{0}^{2} e^{-A \Lambda}\left(p_{\varepsilon}^{(k)} v_{\varepsilon}\right) \cdot\left(\overline{p_{j}^{(k)} v_{\varepsilon}}\right)\right\} \\
& \left.\quad-\operatorname{Re}\left\{W_{0}^{2} e^{-A \Lambda}\left(\partial_{t} p_{j}^{(k)}\right)\left(t, D_{t}, \xi ; \varepsilon\right) v_{\varepsilon}\right) \cdot\left(\overline{p_{j}^{(k)} v_{\varepsilon}}\right)\right\}
\end{aligned}
$$


$(1 \leq k \leq m-1,1 \leq j \leq m-k)$. Therefore, (3.1), (3.3) and (3.6)-(3.8) yield

$$
\begin{aligned}
\partial_{t} \mathscr{E}(t, \xi ; \varepsilon ; A) \leq & m \Lambda_{t}^{-1} e^{-A \Lambda}|\hat{f}(t, \xi)|^{2}-\sum_{j=1}^{m}\left[(A-2 m-1) \Lambda_{t} e^{-A \Lambda}\left|\mathscr{P}_{j} v_{\varepsilon}\right|^{2}\right. \\
& +\Lambda_{t}^{-1} e^{-A \Lambda}\left\{\left|\operatorname{sub} \sigma(P)\left(t, D_{t}, \xi\right) v_{\varepsilon}\right|^{2}+\sum_{k \neq j}\left|\left(\lambda_{j t}-\lambda_{k t}\right) p_{j, k} v_{\varepsilon}\right|^{2} / 4\right. \\
& \left.\left.+\sum_{k=2}^{m}\left|P_{m-k}\left(t, D_{t}, \xi\right) v_{\varepsilon}\right|^{2}+\left|\left(\partial_{t}^{2} \partial_{\tau} p_{j}\right)\left(t, D_{t}, \xi ; \varepsilon\right) v_{\varepsilon}\right|^{2} / 4\right\}\right] \\
& -\sum_{k=1}^{m-1} \sum_{j=1}^{m-k}\left[\left\{(A-2) W_{0}^{2} \Lambda_{t}-2 W_{0}^{3}\right\} e^{-A \Lambda}\left|p_{j}^{(k)} v_{\varepsilon}\right|^{2}\right. \\
& \left.-W_{0}^{2} \Lambda_{t}^{-1} e^{-A \Lambda}\left\{\left|p_{\varepsilon}^{(k)} v_{\varepsilon}\right|^{2}+\left|\left(\partial_{t} p_{j}^{(k)}\right)\left(t, D_{t}, \xi ; \varepsilon\right) v_{\varepsilon}\right|^{2}\right\}\right],
\end{aligned}
$$

since

$$
\begin{aligned}
\left(p_{\varepsilon}-\right. & \left.(i / 2)\left(\partial_{t} \partial_{\tau} p_{\varepsilon}\right)\left(t, D_{t}, \xi\right)\right) v_{\varepsilon} \\
& =P_{\varepsilon} v_{\varepsilon}-s u b \sigma(P)\left(t, D_{t}, \xi\right) v_{\varepsilon}-\sum_{k=2}^{m} P_{m-k}\left(t, D_{t}, \xi\right) v_{\varepsilon}
\end{aligned}
$$

From (D-L), Lemma 2.6 and Corollary 2.7 we have, with some $C, C^{\prime}>0$,

$$
\begin{aligned}
& \min \left\{\min _{s \in \mathscr{R}(\xi / \xi \mid)}|t-s|^{2}, 1\right\}\left|\operatorname{sub} \sigma(P)\left(t, D_{t}, \xi\right) v_{\varepsilon}\right|^{2} \\
& \leq m C^{2} \sum_{j=1}^{m}\left|p_{j} v_{\varepsilon}\right|^{2} \leq C^{\prime}\left(\sum_{j=1}^{m}\left|\mathscr{P}_{j} v_{\varepsilon}\right|^{2}+\sum_{k=1}^{m-1}\left|p_{k}^{(1)} v_{\varepsilon}\right|^{2}\right)
\end{aligned}
$$

for $t \in[0, T], \xi \in \mathbf{R}^{n} \backslash \mathscr{N}$ and $\varepsilon \in E_{0}$. Here and after the constants do not depend on the parameter $A$ unless stated. Indeed, $p^{(1)}(t, \tau, \xi ; \varepsilon)$ is stictly hyperbolic with respect to $\vartheta$ for $t \in[0, \infty)$ and $\varepsilon \in \mathbf{R}$. By Corollary 2.7 with $r=m-1$ and (3.5) we have, with some $C^{\prime \prime}>0$,

$$
\left|\left(\partial_{t} \partial_{\tau} p_{j}\right)\left(t, D_{t}, \xi ; \varepsilon\right) v_{\varepsilon}\right| \leq C^{\prime \prime} \sum_{k=1}^{m-1}\left|p_{k}^{(1)} v_{\varepsilon}\right| .
$$

When $\min \left\{\min _{s \in \mathscr{R}(\xi /|\xi|)}|t-s|, 1\right\} \leq\langle\xi\rangle^{-1 / 2}$, we can not use (3.10). It follows from Corollary 2.7 that 


$$
\begin{aligned}
\operatorname{sub} \sigma(P)(t, \tau, \xi) & =c_{0}(t) \tau^{m-1}+\sum_{k=1}^{m-1} c_{k}(t, \xi) \tau^{m-k-1} \\
& =c_{0}(t) \mathscr{P}_{j}(t, \tau, \xi ; \varepsilon)+\sum_{k=1}^{m-1} \tilde{c}_{k}(t, \xi ; \varepsilon) p_{k}^{(1)}(t, \tau, \xi ; \varepsilon),
\end{aligned}
$$

where $1 \leq j \leq m, \operatorname{deg}_{\xi} c_{k}(t, \xi)=k$ and the $\tilde{c}_{k}(t, \xi ; \varepsilon)$ are functions, determined by Corollary 2.7 , satisfying, with some $C>0$,

$$
\left|\tilde{c}_{k}(t, \xi ; \varepsilon)\right| \leq C\langle\xi\rangle \text { for } t \in[0, T], \xi \in \mathbf{R}^{n} \backslash \mathscr{N} \text { and } \varepsilon \in E_{0} \text {. }
$$

This gives

$$
\left|\operatorname{sub} \sigma(P)\left(t, D_{t}, \xi\right) v_{\varepsilon}\right|^{2} \leq C\left(\left|\mathscr{P}_{j} v_{\varepsilon}\right|^{2}+\langle\xi\rangle^{2} \sum_{k=1}^{m-1}\left|p_{k}^{(1)} v_{\varepsilon}\right|^{2}\right)
$$

for $t \in[0, T], \xi \in \mathbf{R}^{n} \backslash \mathscr{N}$ and $\varepsilon \in E_{0}$. We have also, with some $C>0$,

$$
\begin{aligned}
\mid\left(\lambda_{j}-\right. & \left.\lambda_{k}\right)\left.p_{j, k} v_{\varepsilon}\right|^{2} \\
& \leq\left|\left\{\mathscr{P}_{j}-\mathscr{P}_{k}+(i / 2)\left(\left(\partial_{t} \partial_{\tau} p_{j}\right)\left(t, D_{t}, \xi ; \varepsilon\right)-\left(\partial_{t} \partial_{\tau} p_{k}\right)\left(t, D_{t}, \xi ; \varepsilon\right)\right)\right\} v_{\varepsilon}\right|^{2} \\
& \leq 3\left\{\left|\mathscr{P}_{j} v_{\varepsilon}\right|^{2}+\left|\mathscr{P}_{k} v_{\varepsilon}\right|^{2}+C \sum_{l=1}^{m-1}\left|p_{l}^{(1)} v_{\varepsilon}\right|^{2}\right\}
\end{aligned}
$$

$$
\begin{aligned}
& \sum_{k=2}^{m}\left|P_{m-k}\left(t, D_{t}, \xi\right) v_{\varepsilon}\right|^{2} \leq C \sum_{k=1}^{m-1} \sum_{j=1}^{m-k}\left|p_{j}^{(k)} v_{\varepsilon}\right|^{2} \\
& \left|\left(\partial_{t}^{2} \partial_{\tau} p_{j}\right)\left(t, D_{t}, \xi ; \varepsilon\right) v_{\varepsilon}\right|^{2}
\end{aligned}
$$$$
\leq C \sum_{k=1}^{m-1}\left|p_{k}^{(1)} v_{\varepsilon}\right|^{2}+C \sum_{k=1}^{m}\left|\lambda_{k t t}(t, \xi ; \varepsilon)\right|^{2}|\xi|^{-2} \sum_{l=1}^{m-1}\left|p_{l}^{(1)} v_{\varepsilon}\right|^{2},
$$

$$
\begin{aligned}
& W_{0}^{2} \Lambda_{t}^{-1} e^{-A \Lambda}\left|p_{\varepsilon}^{(1)} v_{\varepsilon}\right|^{2} \\
& =W_{0}^{2} \Lambda_{t}^{-1} e^{-A \Lambda}\left|\sum_{j=1}^{m}\left(\mathscr{P}_{j}+(i / 2)\left(\partial_{t} \partial_{\tau} p_{j}\right)\left(t, D_{t}, \xi ; \varepsilon\right)\right) v_{\varepsilon}\right|^{2} \\
& \leq C W_{0}^{2} \Lambda_{t}^{-1} e^{-A \Lambda}\left\{\sum_{j=1}^{m}\left|\mathscr{P}_{j} v_{\varepsilon}\right|^{2}+\sum_{k=1}^{m-1}\left|p_{k}^{(1)} v_{\varepsilon}\right|^{2}\right\}
\end{aligned}
$$




$$
\begin{gathered}
W_{0}^{2} \Lambda_{t}^{-1} e^{-A \Lambda}\left|\left(\partial_{t} p_{\varepsilon}^{(1)}\right)\left(t, D_{t}, \xi ; \varepsilon\right) v_{\varepsilon}\right|^{2} \leq C W_{0}^{2} \Lambda_{t}^{-1} e^{-A \Lambda} \sum_{k=1}^{m-1}\left|p_{k}^{(1)} v_{\varepsilon}\right|^{2} \\
W_{0}^{2} \Lambda_{t}^{-1} e^{-A \Lambda}\left\{\left|p_{\varepsilon}^{(k)} v_{\varepsilon}\right|^{2}+\left.\left(\partial_{t} p_{j}^{(k)}\right)\left(t, D_{t}, \xi ; \varepsilon\right) v_{\varepsilon}\right|^{2}\right\} \\
\leq C W_{0}^{2} \Lambda_{t}^{-1} e^{-A \Lambda}\left\{\sum_{l=1}^{m-k+1}\left|p_{l}^{(k-1)} v_{\varepsilon}\right|^{2}+\sum_{l=1}^{m-k}\left|p_{l}^{(k)} v_{\varepsilon}\right|^{2}\right\}
\end{gathered}
$$

$$
\text { for } 2 \leq k \leq m-1 \text { and } 1 \leq j \leq m-k
$$

for $t \in[0, T], \quad \xi \in \mathbf{R}^{n} \backslash \mathscr{N}$ and $\varepsilon \in E_{0}$. Indeed, (3.12), (3.13) and (3.15) follow from Corollary 2.7, applying the argument as in $(3.10)$, since $p^{(k)}(t, \tau, \xi ; \varepsilon)$ $(1 \leq k \leq m-1)$ are strictly hyperbolic with respect to $\vartheta$. We have

$$
\begin{aligned}
\partial_{t}^{2} \partial_{\tau} p_{j}(t, \tau, \xi ; \varepsilon)= & -\sum_{k \neq j} \sum_{l \neq j, k} \lambda_{l t t}(t, \xi ; \varepsilon) p_{j, k, l}(t, \tau, \xi ; \varepsilon) \\
& +\sum_{k \neq j} \sum_{l \neq j, k} \sum_{i \neq j, k, l} \lambda_{l t}(t, \xi ; \varepsilon) \lambda_{i t}(t, \xi ; \varepsilon) p_{j, k, l, i}(t, \tau, \xi ; \varepsilon)
\end{aligned}
$$

By (3.5) and the same argument as in (3.10) we obtain (3.14), (3.16) and (3.17). Let us estimate $\Lambda_{t}^{-1} e^{-A \Lambda}\left|\operatorname{sub} \sigma(P)\left(t, D_{t}, \xi\right) v_{\varepsilon}\right|^{2}$. First assume that $\min \left\{\min _{s \in \mathscr{R}(\xi / \mid \xi) \mid}|t-s|, 1\right\} \leq\langle\xi\rangle^{-1 / 2}$. Then we have

$$
W_{0}(t, \xi) \geq\langle\xi\rangle^{1 / 2} / \sqrt{2}
$$

Therefore, from (3.11) we have

$$
\begin{aligned}
& \Lambda_{t}^{-1} e^{-A \Lambda}\left|\operatorname{sub} \sigma(P)\left(t, D_{t}, \xi\right) v_{\varepsilon}\right|^{2} \\
& \quad \leq C \Lambda_{t}^{-1} e^{-A \Lambda}\left(\left|\mathscr{P}_{j} v_{\varepsilon}\right|^{2}+4 W_{0}^{4} \sum_{k=1}^{m-1}\left|p_{k}^{(1)} v_{\varepsilon}\right|^{2}\right) \\
& \quad \leq C \Lambda_{t}^{-1} e^{-A \Lambda}\left|\mathscr{P}_{j} v_{\varepsilon}\right|^{2}+4 C \sum_{k=1}^{m-1} W_{0}^{2} \Lambda_{t} e^{-A \Lambda}\left|p_{k}^{(1)} v_{\varepsilon}\right|^{2}
\end{aligned}
$$

for $t \in[0, T], \xi \in \mathbf{R}^{n} \backslash \mathscr{N}$ and $\varepsilon \in E_{0}$. Next assume that $\min \left\{\min _{s \in \mathscr{R}(\xi /|\xi|)}|t-s|, 1\right\}$ $\geq\langle\xi\rangle^{-1 / 2}$. Then we have

$$
W_{0}(t, \xi) \geq\left(\sqrt{2} \min \left\{\min _{s \in \mathscr{R}(\xi / \xi \mid)}|t-s|, 1\right\}\right)^{-1} .
$$


This, together with (3.10), yields

$$
\begin{aligned}
\Lambda_{t}^{-1} e^{-A \Lambda}\left|\operatorname{sub} \sigma(P)\left(t, D_{t}, \xi\right) v_{\varepsilon}\right|^{2} & \\
& \leq 2 C^{\prime} W_{0}^{2} \Lambda_{t}^{-1} e^{-A \Lambda}\left(\sum_{j=1}^{m}\left|\mathscr{P}_{j} v_{\varepsilon}\right|^{2}+\sum_{k=1}^{m-1}\left|p_{k}^{(1)} v_{\varepsilon}\right|^{2}\right) \\
& \leq 2 C^{\prime} \Lambda_{t} e^{-A \Lambda} \sum_{j=1}^{m}\left|\mathscr{P}_{j} v_{\varepsilon}\right|^{2}+2 C^{\prime} W_{0}^{2} \Lambda_{t} e^{-A \Lambda} \sum_{k=1}^{m-1}\left|p_{k}^{(1)} v_{\varepsilon}\right|^{2}
\end{aligned}
$$

for $t \in[0, T], \xi \in \mathbf{R}^{n} \backslash \mathscr{N}$ and $\varepsilon \in E_{0}$. Thus, by (3.18) and (3.19) we have

$$
\begin{aligned}
& \Lambda_{t}^{-1} e^{-A \Lambda}\left|\operatorname{sub} \sigma(P)\left(t, D_{t}, \xi\right) v_{\varepsilon}\right|^{2} \\
& \quad \leq C \Lambda_{t} e^{-A \Lambda} \sum_{j=1}^{m}\left|\mathscr{P}_{j} v_{\varepsilon}\right|^{2}+C W_{0}^{2} \Lambda_{t} e^{-A \Lambda} \sum_{k=1}^{m-1}\left|p_{k}^{(1)} v_{\varepsilon}\right|^{2}
\end{aligned}
$$

for $t \in[0, T], \xi \in \mathbf{R}^{n} \backslash \mathscr{N}$ and $\varepsilon \in E_{0}$. It follows from (3.12) and the definition of $W_{1}(t, \xi ; \varepsilon)$ that

$$
\begin{aligned}
\Lambda_{t}^{-1} e^{-A \Lambda}\left|\left(\lambda_{j t}-\lambda_{k t}\right) p_{j, k} v_{\varepsilon}\right|^{2} & \\
& \leq 2 W_{1} e^{-A \Lambda}\left(\left|\lambda_{j}-\lambda_{k}\right|^{2}+1\right)\left|p_{j, k} v_{\varepsilon}\right|^{2} \\
& \leq 6 \Lambda_{t} e^{-A \Lambda}\left(\left|\mathscr{P}_{j} v_{\varepsilon}\right|^{2}+\left|\mathscr{P}_{k} v_{\varepsilon}\right|^{2}\right)+C^{\prime} W_{0}^{2} \Lambda_{t} e^{-A \Lambda} \sum_{k=1}^{m}\left|p_{k}^{(1)} v_{\varepsilon}\right|^{2}
\end{aligned}
$$

for $t \in[0, T], \xi \in \mathbf{R}^{n} \backslash \mathscr{N}$ and $\varepsilon \in E_{0}$. From (3.13)-(3.17) we have

$$
\Lambda_{t}^{-1} e^{-A \Lambda} \sum_{k=2}^{m}\left|P_{m-k}\left(t, D_{t}, \xi\right) v_{\varepsilon}\right|^{2} \leq C W_{0}^{2} \Lambda_{t} e^{-A \Lambda} \sum_{k=1}^{m-1} \sum_{j=1}^{m-k}\left|p_{j}^{(k)} v_{\varepsilon}\right|^{2}
$$

$$
\Lambda_{t}^{-1} e^{-A \Lambda}\left|\left(\partial_{t}^{2} \partial_{\tau} p_{j}\right)\left(t, D_{t}, \xi ; \varepsilon\right) v_{\varepsilon}\right|^{2} \leq 2 C W_{0}^{2} \Lambda_{t} e^{-A \Lambda} \sum_{k=1}^{m-1}\left|p_{k}^{(1)} v_{\varepsilon}\right|^{2}
$$

$$
W_{0}^{2} \Lambda_{t}^{-1} e^{-A \Lambda}\left|p_{\varepsilon}^{(1)} v_{\varepsilon}\right|^{2} \leq C \Lambda_{t} e^{-A \Lambda} \sum_{j=1}^{m}\left|\mathscr{P}_{j} v_{\varepsilon}\right|^{2}+C W_{0}^{2} \Lambda_{t} e^{-A \Lambda} \sum_{k=1}^{m-1}\left|p_{k}^{(1)} v_{\varepsilon}\right|^{2}
$$

$$
W_{0}^{2} \Lambda_{t}^{-1} e^{-A \Lambda}\left|\left(\partial_{t} p_{j}^{(1)}\right)\left(t, D_{t}, \xi ; \varepsilon\right) v_{\varepsilon}\right|^{2} \leq C W_{0}^{2} \Lambda_{t} e^{-A \Lambda} \sum_{k=1}^{m-1}\left|p_{k}^{(1)} v_{\varepsilon}\right|^{2}
$$


On the Cauchy problem for a class of hyperbolic operators

$$
\begin{aligned}
& W_{0}^{2} \Lambda_{t}^{-1} e^{-A \Lambda}\left\{\left|p_{\varepsilon}^{(k)} v_{\varepsilon}\right|+\left|\left(\partial_{t} p_{j}^{(k)}\right)\left(t, D_{t}, \xi ; \varepsilon\right) v_{\varepsilon}\right|^{2}\right\} \\
& \leq C W_{0}^{2} \Lambda_{t} e^{-A \Lambda}\left\{\sum_{l=1}^{m-k+1}\left|p_{l}^{(k-1)} v_{\varepsilon}\right|^{2}+\sum_{l=1}^{m-k}\left|p_{l}^{(k)} v_{\varepsilon}\right|^{2}\right\} \\
& \quad \text { for } 2 \leq k \leq m-1 \text { and } 1 \leq j \leq m-k
\end{aligned}
$$

for $t \in[0, T], \xi \in \mathbf{R}^{n} \backslash \mathscr{N}$ and $\varepsilon \in E_{0}$, since $\left|\lambda_{k t t}\right|^{2}|\xi|^{-2} \leq W_{1}^{2}$. So it follows from (3.9) and (3.20)-(3.26) that there is $A_{0} \geq 1$ satisfying

$$
\partial_{t} \mathscr{E}(t, \xi ; \varepsilon ; A) \leq m \Lambda_{t}^{-1} e^{-A \Lambda}|\hat{f}(t, \xi)|^{2}
$$

for $A \geq A_{0}, t \in[0, T], \xi \in \mathbf{R}^{n} \backslash \mathscr{N}$ and $\varepsilon \in E_{0}$. This gives

$$
\mathscr{E}(t, \xi ; \varepsilon ; A) \leq \mathscr{E}(0, \xi ; \varepsilon ; A)+m \int_{0}^{t}|\hat{f}(s, \xi)|^{2} d s
$$

for $A \geq A_{0}, t \in[0, T], \xi \in \mathbf{R}^{n} \backslash \mathscr{N}$ and $\varepsilon \in E_{0}$. From (3.4) we have

$$
1 \geq e^{-A \Lambda(t, \xi ; \varepsilon)} \geq e^{-A C_{T}}\langle\xi\rangle^{-A C_{T}}
$$

for $t \in[0, T], \xi \in \mathbf{R}^{n} \backslash \mathscr{N}$ and $\varepsilon \in E_{0}$.

Lemma 3.1. For a fixed $T>0$ there are $c>0$ and $C_{A}>0$, which depends on A, such that

$$
c \mathscr{E}(t, \xi ; \varepsilon ; A) \leq \sum_{k=0}^{m-1}\langle\xi\rangle^{2 k}\left|D_{t}^{m-1-k} v_{\varepsilon}\right|^{2} \leq C_{A}\langle\xi\rangle^{2+A C_{T}} \mathscr{E}(t, \xi ; \varepsilon ; A)
$$

for $A \geq 1, \quad t \in[0, T], \quad \xi \in \mathbf{R}^{n}$ and $\varepsilon \in[0,1]$. In particular, we have $v_{\varepsilon}(t, \xi) \in$ $C^{m-1}\left([0, \infty) ; \mathscr{S}^{\prime}\left(\mathbf{R}_{\xi}^{n}\right)\right) \quad$ and $u_{\varepsilon}(t, x)\left(\equiv \mathscr{F}_{\xi}^{-1}\left[v_{\varepsilon}(t, \xi)\right](x)\right) \in C^{m-1}\left([0, \infty) ; H^{\infty}\left(\mathbf{R}^{n}\right)\right)$ for $\varepsilon \in E_{0}$.

Proof. We can write

$$
D_{t}^{m-1} v_{\varepsilon}(t, \xi)=\mathscr{P}_{1}\left(t, D_{t}, \xi ; \varepsilon\right) v_{\varepsilon}+\sum_{j=1}^{m-1} c_{j}(t, \xi ; \varepsilon) D_{t}^{m-1-j} v_{\varepsilon}+(i / 2) \partial_{t} \partial_{\tau} p_{1}\left(t, D_{t}, \xi ; \varepsilon\right) v_{\varepsilon}
$$

where $c_{j}(t, \xi ; \varepsilon) \quad(1 \leq j \leq m-1)$ satisfy $\left|c_{j}(t, \xi ; \varepsilon)\right| \leq C|\xi|^{j}$ for $\varepsilon \in[0,1]$. So Corollary 2.7 gives

$$
\left|D_{t}^{m-1} v_{\varepsilon}(t, \xi)\right| \leq\left|\mathscr{P}_{1}\left(t, D_{t}, \xi ; \varepsilon\right) v_{\varepsilon}\right|+C\langle\xi\rangle \sum_{j=1}^{m-1}\left|p_{j}^{(1)}\left(t, D_{t}, \xi ; \varepsilon\right) v_{\varepsilon}\right|
$$


Similarly, we have

$$
\langle\xi\rangle^{k}\left|D_{t}^{m-1-k} v_{\varepsilon}\right| \leq C\left(|\xi| \sum_{j=1}^{m-1}\left|p_{j}^{(1)}\left(t, D_{t}, \xi ; \varepsilon\right) v_{\varepsilon}\right|+\sum_{j=1}^{m-k}\left|p_{j}^{(k)} v_{\varepsilon}\right|\right)
$$

$(1 \leq k \leq m-1)$. Therefore, we have

$$
\begin{aligned}
\sum_{k=0}^{m-1}\langle\xi\rangle^{2 k}\left|D_{t}^{m-1-k} v_{\varepsilon}\right|^{2} & \leq C_{A}\langle\xi\rangle^{2+A C_{T}} e^{-A \Lambda}\left(\left|\mathscr{P}_{1} v_{\varepsilon}\right|^{2}+\sum_{k=1}^{m-1} \sum_{j=1}^{m-k} W_{0}^{2}\left|p_{j}^{(k)} v_{\varepsilon}\right|^{2}\right) \\
& \leq C_{A}\langle\xi\rangle^{2+A C_{T}} \mathscr{E}(t, \xi ; \varepsilon ; A)
\end{aligned}
$$

for $A \geq 1, t \in[0, T], \xi \in \mathbf{R}^{n}$ and $\varepsilon \in[0,1]$, where $C_{A}(>0)$ depends on $A$. It is obvious that, with some $C, C^{\prime}>0$,

$$
\begin{aligned}
\mathscr{E}(t, \xi ; \varepsilon ; A) & \leq \sum_{j=1}^{m}\left|\mathscr{P}_{j} v_{\varepsilon}\right|^{2}+\sum_{k=1}^{m-1} \sum_{j=1}^{m-k} C\langle\xi\rangle\left|p_{j}^{(k)} v_{\varepsilon}\right|^{2} \\
& \leq C^{\prime} \sum_{k=0}^{m-1}\langle\xi\rangle^{2 k}\left|D_{t}^{m-1-k} v_{\varepsilon}\right|^{2}
\end{aligned}
$$

for $t \in[0, T], \xi \in \mathbf{R}^{n}$ and $\varepsilon \in[0,1]$, since $W_{0}(t, \xi)^{2} \leq C\langle\xi\rangle$. This, together with (3.29), proves the lemma.

Fix $T>0$ and put

$$
E_{k, l}[u](t)=\sum_{\mu=0}^{k}\left\|\left\langle D_{x}\right\rangle^{l+k-\mu} D_{t}^{\mu} u(t, x)\right\|_{L^{2}}^{2}
$$

where $\quad k \geq m-1, \quad l \in \mathbf{R}, \quad u(t, x) \in C^{k}\left([0, T] ; H^{\infty}\left(\mathbf{R}_{x}^{n}\right)\right) \quad$ and $\quad\|u(t, x)\|_{L^{2}}=$ $\left(\int_{\mathbf{R}^{n}}|u(t, x)|^{2} d x\right)^{1 / 2}$. It follows from (3.27), (3.28) and Plancherel's theorem that

$$
\begin{aligned}
E_{m-1, l}\left[u_{\varepsilon}\right](t) \leq C_{A_{0}}\{ & \sum_{v=0}^{m-1}\left\|\left\langle D_{x}\right\rangle^{l+m+A_{0} C_{T} / 2-v} u_{v}(x)\right\|_{L^{2}}^{2} \\
& \left.+\int_{0}^{t}\left\|\left\langle D_{x}\right\rangle^{l+1+A_{0} C_{T} / 2} f(s, x)\right\|_{L^{2}}^{2} d s\right\}
\end{aligned}
$$

for $l \in \mathbf{R}, t \in[0, T]$ and $\varepsilon \in E_{0}$. 
LemmA 3.2. For $\varepsilon \in E_{0}$ we have $u_{\varepsilon}(t, x) \in C^{\infty}\left([0, \infty) ; H^{\infty}\left(\mathbf{R}^{n}\right)\right)$. Fix $T>0$. Then, for any $k \in \mathbf{Z}_{+}$with $k \geq m-1$ there is $C_{k}>0$ such that

$$
\begin{aligned}
E_{k, l}\left[u_{\varepsilon}\right](t) \leq C_{k}\{ & \sum_{v=0}^{m-1}\left\|\left\langle D_{x}\right\rangle^{l+k+1+A_{0} C_{T} / 2-v} u_{v}(x)\right\|_{L^{2}}^{2} \\
& +\int_{0}^{t}\left\|\left\langle D_{x}\right\rangle^{l+k+2-m+A_{0} C_{T} / 2} f(s, x)\right\|_{L^{2}}^{2} d s \\
& \left.+\sum_{\mu=0}^{k-m}\left\|\left\langle D_{x}\right\rangle^{l+k-m-\mu} D_{t}^{\mu} f(t, x)\right\|_{L^{2}}^{2}\right\}
\end{aligned}
$$

for $l \in \mathbf{R}, t \in[0, T]$ and $\varepsilon \in E_{0}$, where $\sum_{\mu=0}^{k-m} \cdots=0$ if $k=m-1$.

Proof. By (3.30) (3.31) with $k=m-1$ is valid. Let $K \geq m$, and assume that $u_{\varepsilon}(t, x) \in C^{K-1}\left([0, T] ; H^{\infty}\left(\mathbf{R}^{n}\right)\right)$ and that $(3.31)$ is valid if $(m-1 \leq) k \leq K-1$. Write

$$
P_{\varepsilon}(t, \tau, \xi)=\tau^{m}+\sum_{j=1}^{m} a_{j, \varepsilon}(t, \xi) \tau^{m-j}
$$

Then we have

$$
\begin{aligned}
D_{t}^{K} v_{\varepsilon}(t, \xi)= & -\sum_{j=1}^{m} \sum_{\mu=0}^{K-m}\left(\begin{array}{c}
K-m \\
\mu
\end{array}\right)\left(D_{t}^{K-m-\mu} a_{j, \varepsilon}(t, \xi)\right) D_{t}^{m-j+\mu} v_{\varepsilon}(t, \xi) \\
& +D_{t}^{K-m} \hat{f}(t, \xi) .
\end{aligned}
$$

Since the right-hand side of (3.32) belongs to $C\left([0, T] ; \mathscr{F}^{-1}\left(H^{\infty}\left(\mathbf{R}^{n}\right)\right)\right)$, we have $u_{\varepsilon} \in C^{K}\left([0, T] ; H^{\infty}\left(\mathbf{R}^{n}\right)\right)$. Moreover, we have

$$
\begin{aligned}
& \left\|\left\langle D_{x}\right\rangle^{l} D_{t}^{K} u_{\varepsilon}(t, x)\right\|_{L^{2}\left(\mathbf{R}_{x}^{n}\right)}^{2} \\
& \quad \leq C_{K}\left\{\sum_{\mu=0}^{K-1}\left\|\left\langle D_{x}\right\rangle^{l+K-\mu} D_{t}^{\mu} u_{\varepsilon}(t, x)\right\|_{L^{2}\left(\mathbf{R}_{x}^{n}\right)}^{2}+\left\|\left\langle D_{x}\right\rangle^{l} D_{t}^{K-m} f(t, x)\right\|_{L^{2}\left(\mathbf{R}_{x}^{n}\right)}^{2}\right\}
\end{aligned}
$$

for $l \in \mathbf{R}, t \in[0, T]$ and $\varepsilon \in E_{0}$. Therefore, (3.31) is valid for $k=K$.

Put $u(t, x)=u_{0}(t, x)$ and $u_{j}(t, x)=u_{\varepsilon_{j}}(t, x)$. Applying the same argument as in $\S 3$ of [16], we can prove that

$$
D_{t}^{k} D_{x}^{\alpha} u_{j}(t, x) \rightarrow D_{t}^{k} D_{x}^{\alpha} u(t, x) \quad \text { uniformly in }[0, T] \times \mathbf{R}^{n} \text { as } j \rightarrow \infty .
$$


Denote by $K_{j,\left(t_{0}, x^{0}\right)}^{ \pm}$the generalized flows for $p_{\varepsilon_{j}}(t, \tau, \xi)$. Then it follows from $\S 3$ of [13] (or [15]) that for any $\left(t_{0}, x^{0}\right) \in(0, \infty) \times \mathbf{R}^{n}$ and any neighborhood $V$ of $K_{\left(t_{0}, x^{0}\right)}^{-} \cap\{t \geq 0\}$ there is $J \in \mathbf{N}$ such that

$$
K_{j,\left(t_{0}, x^{0}\right)}^{-} \cap\{t \geq 0\} \subset V \quad \text { if } j \geq J .
$$

Since $P_{\varepsilon_{j}}\left(t, D_{t}, D_{x}\right)$ is strictly hyperbolic with respect to $\vartheta$, we can show that $\left(t_{0}, x^{0}\right) \notin \operatorname{supp} w$ if $j \in \mathbf{N},\left(t_{0}, x^{0}\right) \in(0, \infty) \times \mathbf{R}^{n}, w(t, x) \in C^{\infty}\left([0, \infty) \times \mathbf{R}^{n}\right)$, $\operatorname{supp} P_{\varepsilon_{j}}\left(t, D_{t}, D_{x}\right) w(t, x) \cap K_{j,\left(t_{0}, x^{0}\right)}^{-} \cap\{t \geq 0\}=\varnothing$ and $\{0\} \times\left(\bigcup_{k=0}^{m-1} \operatorname{supp}\left(D_{t}^{k} w\right)(0\right.$, $x)) \cap K_{j,\left(t_{0}, x^{0}\right)}^{-}=\varnothing$ (see, e.g., [9]). So we can repeat the same arguments as in the end of $\S 3$ of [16] and prove Theorem 1.2.

\section{Proof of Theorem 1.3}

In this section we assume that the hypotheses of Theorem 1.3 are fulfilled and we shall prove Theorem 1.3. We shall change the definitions of $E_{0}, \mathscr{N}, W_{0}, W_{1}$, $\Lambda$ and $\mathscr{E}(t, \xi ; \varepsilon ; A)$. Let $\left\{\varepsilon_{j}\right\}_{j=1,2, \ldots}$ be a sequence satisfying $\varepsilon_{j} \in(0,1] \backslash\left(\mathscr{N}_{2}^{0}(p) \cup\right.$ $\left.\mathscr{N}_{1}^{0}\left(p^{(1)}\right)\right)$ and $\varepsilon_{j} \downarrow 0$ as $j \rightarrow \infty$, where $\mathscr{N}_{2}^{0}(p)$ and $\mathscr{N}_{1}^{0}\left(p^{(1)}\right.$ are as in Lemma 2.3. Put

$$
\begin{aligned}
& E_{0}=\left\{\varepsilon_{j} ; j=1,2, \ldots\right\} \cup\{0\} \\
& \mathscr{N}=\bigcup_{j=1}^{\infty}\left(\mathscr{N}_{2}\left(\varepsilon_{j} ; p\right) \cup \mathscr{N}_{1}\left(\varepsilon_{j} ; p^{(1)}\right)\right) \cup \mathscr{N}_{2}(0 ; p) \cup \mathscr{N}_{1}\left(0 ; p^{(1)}\right) \cup\{0\},
\end{aligned}
$$

where $\mathscr{N}_{2}(\varepsilon ; p)$ and $\mathscr{N}_{1}\left(\varepsilon ; p^{(1)}\right)$ are as in Lemma 2.3. We note that $\mu_{n}(\mathscr{N})=0$. Consider the Cauchy problem $(\mathrm{CP})_{\varepsilon}$ and (3.1) with $m=3$. Fix $T>0$. Define

$$
\begin{aligned}
W_{0}(t, \xi ; \varepsilon)= & \sum_{s \in \mathscr{R}(\xi / \xi \xi) \cap[0, T+1]}\langle\xi\rangle^{2 / 3} / \sqrt{(t-s)^{2}\langle\xi\rangle^{4 / 3}+1} \\
& +\sum_{1 \leq j<k \leq 3} \sqrt{\left(\partial_{t}\left(\lambda_{j}(t, \xi ; \varepsilon)-\lambda_{k}(t, \xi ; \varepsilon)\right)\right)^{2}+1} \\
& \times 1 / \sqrt{\left(\lambda_{j}(t, \xi ; \varepsilon)-\lambda_{k}(t, \xi ; \varepsilon)\right)^{2}+1}+1, \\
W_{1}(t, \xi ; \varepsilon)= & \sum_{1 \leq j<k \leq 3}\left|\partial_{t}^{2}\left(\lambda_{j}(t, \xi ; \varepsilon)-\lambda_{k}(t, \xi ; \varepsilon)\right)\right| /\left(\left|\partial_{t}\left(\lambda_{j}(t, \xi ; \varepsilon)-\lambda_{k}(t, \xi ; \varepsilon)\right)\right|+1\right) \\
& +\left|\partial_{t}\left(\lambda_{2}^{(1)}(t, \xi ; \varepsilon)-\lambda_{1}^{(1)}(t, \xi ; \varepsilon)\right)\right| /\left(\left|\lambda_{2}^{(1)}(t, \xi ; \varepsilon)-\lambda_{1}^{(1)}(t, \xi ; \varepsilon)\right|+1\right), \\
\Lambda(t, \xi ; \varepsilon)= & \int_{0}^{t}\left(W_{0}(s, \xi ; \varepsilon)+W_{1}(s, \xi ; \varepsilon)\right) d s
\end{aligned}
$$


for $(t, \xi, \varepsilon) \in[0, T] \times\left(\mathbf{R}^{n} \backslash \mathscr{N}\right) \times E_{0}$. It is easy to see that

$$
\begin{aligned}
\left|\partial_{t} W_{0}(t, \xi ; \varepsilon)\right| & \leq W_{0}(t, \xi ; \varepsilon)\left(W_{0}(t, \xi ; \varepsilon)+\sqrt{2} W_{1}(t, \xi ; \varepsilon)\right) \\
& \leq 3 W_{0}(t, \xi ; \varepsilon) \partial_{t} \Lambda(t, \xi ; \varepsilon)
\end{aligned}
$$

for $(t, \xi, \varepsilon) \in[0, T] \times\left(\mathbf{R}^{n} \backslash \mathscr{N}\right) \times E_{0}$. By Lemmas 2.3 and 2.4 there is $C_{T}>0$ satisfying

$$
0 \leq \Lambda(t, \xi ; \varepsilon) \leq C_{T}(\log \langle\xi\rangle+1)
$$

for $(t, \xi, \varepsilon) \in[0, T] \times\left(\mathbf{R}^{n} \backslash \mathscr{N}\right) \times E_{0}$, since

$$
\partial_{t} \log \left(\sqrt{(t-s)^{2}\langle\xi\rangle^{4 / 3}+1}+(t-s)\langle\xi\rangle^{2 / 3}\right)=\langle\xi\rangle^{2 / 3} / \sqrt{(t-s)^{2}\langle\xi\rangle^{4 / 3}+1} .
$$

For $(t, \xi, \varepsilon) \in[0, T] \times\left(\mathbf{R}^{n} \backslash \mathscr{N}\right) \times E_{0}$ and $A \geq 1$ we define

$$
\mathscr{E}(t, \xi ; \varepsilon ; A)=\sum_{j=1}^{3} e^{-A \Lambda}\left|\mathscr{P}_{j} v_{\varepsilon}\right|^{2}+\sum_{j=1}^{2} W_{0}^{2} e^{-A \Lambda}\left|p_{j}^{(1)} v_{\varepsilon}\right|^{2}+W_{0}^{4} e^{-A \Lambda}\left|v_{\varepsilon}\right|^{2}
$$

Here we write $\Lambda=\Lambda(t, \xi ; \varepsilon), \mathscr{P}_{j}=\mathscr{P}_{j}\left(t, D_{t}, \xi ; \varepsilon\right), p_{j}^{(1)}=p_{j}^{(1)}\left(t, D_{t}, \xi ; \varepsilon\right), v_{\varepsilon}=v_{\varepsilon}(t, \xi)$ and $W_{0}=W_{0}(t, \xi ; \varepsilon)$. Let $(t, \xi) \in[0, T] \times\left(\mathbf{R}^{n} \backslash \mathscr{N}\right)$ and $\varepsilon \in E_{0}$. It is obvious that

$$
\begin{aligned}
D_{t} \mathscr{E}(t, \xi ; \varepsilon ; A)= & i \sum_{j=1}^{3}\left[A \Lambda_{t} e^{-A \Lambda}\left|\mathscr{P}_{j} v_{\varepsilon}\right|^{2}+2 \operatorname{Im}\left\{e^{-A \Lambda}\left(D_{t} \mathscr{P}_{j} v_{\varepsilon}\right) \cdot\left(\overline{\mathscr{P}_{j} v_{\varepsilon}}\right)\right\}\right] \\
+ & i \sum_{j=1}^{2}\left[\left(A W_{0}^{2} \Lambda_{t}-2 W_{0} W_{0 t}\right) e^{-A \Lambda}\left|p_{j}^{(1)} v_{\varepsilon}\right|^{2}\right. \\
& \left.+2 \operatorname{Im}\left\{W_{0}^{2} e^{-A \Lambda}\left(D_{t} p_{j}^{(1)} v_{\varepsilon}\right) \cdot\left(\overline{p_{j}^{(1)} v_{\varepsilon}}\right)\right\}\right] \\
+ & i\left[\left(A W_{0}^{4} \Lambda_{t}-4 W_{0}^{3} W_{0 t}\right) e^{-A \Lambda}\left|v_{\varepsilon}\right|^{2}\right. \\
& \left.+2 \operatorname{Im}\left\{W_{0}^{4} e^{-A \Lambda}\left(D_{t} v_{\varepsilon}\right) \cdot \overline{v_{\varepsilon}}\right\}\right]
\end{aligned}
$$

where $\Lambda_{t}=\partial_{t} \Lambda(t, \xi ; \varepsilon)$ and $W_{0 t}=\partial_{t} W_{0}(t, \xi ; \varepsilon)$. Since the $\lambda_{j}(t, \xi ; \varepsilon)$ and the $\lambda_{j}^{(1)}(t, \xi ; \varepsilon)$ are real-valued, $\partial_{t} \partial_{\tau} p_{\varepsilon}(t, \tau, \xi)=\partial_{t} \partial_{\tau} p(t, \tau, \xi)$ and $\partial_{t}^{2} \partial_{\tau}^{2} p_{\varepsilon}(t, \tau, \xi)=$ $\partial_{t}^{2} \partial_{\tau}^{2} p(t, \tau, \xi)$, it follows from (2.20) and (2.22) that

$$
\begin{aligned}
\operatorname{Im}\left\{e^{-A \Lambda}\left(D_{t} \mathscr{P}_{j} v_{\varepsilon}\right) \cdot\left(\overline{\mathscr{P}_{j} v_{\varepsilon}}\right)\right\} \\
\quad=\operatorname{Im}\left\{e^{-A \Lambda}\left(\left(D_{t}-\lambda_{j}\right) \mathscr{P}_{j} v_{\varepsilon}\right) \cdot\left(\overline{\mathscr{P}_{j} v_{\varepsilon}}\right)\right\} \\
\quad=\operatorname{Im}\left\{e^{-A \Lambda}\left(\left(p_{\varepsilon}-(i / 2)\left(\partial_{t} \partial_{\tau} p\right)\left(t, D_{t}, \xi\right)\right) v_{\varepsilon}\right) \cdot\left(\overline{\mathscr{P}_{j} v_{\varepsilon}}\right)\right\}
\end{aligned}
$$




$$
\begin{aligned}
& -\operatorname{Im}\left\{e^{-A \Lambda}\left(\left(\partial_{t}^{2} \partial_{\tau}^{2} p\right)\left(t, D_{t}, \xi\right) v_{\varepsilon}\right) \cdot\left(\overline{\mathscr{P}_{j} v_{\varepsilon}}\right)\right\} / 6 \\
& -\operatorname{Re}\left\{e^{-A \Lambda} \sum_{k \neq j}\left(\lambda_{j t}-\lambda_{k t}\right)\left(p_{j, k} v_{\varepsilon}\right) \cdot\left(\overline{\mathscr{P}_{j} v_{\varepsilon}}\right)\right\} / 2 \\
& +\operatorname{Im}\left\{e^{-A \Lambda} \sum_{k \neq j}\left(\lambda_{j t t}-\lambda_{k t t}\right) v_{\varepsilon} \cdot\left(\overline{\mathscr{P}_{j} v_{\varepsilon}}\right)\right\} / 6,
\end{aligned}
$$

$$
\begin{aligned}
\operatorname{Im}\left\{W_{0}^{2} e^{-A \Lambda}\left(D_{t} p_{j}^{(1)} v_{\varepsilon}\right) \cdot\left(\overline{p_{j}^{(1)} v_{\varepsilon}}\right)\right\} \\
=\operatorname{Im}\left\{W_{0}^{2} e^{-A \Lambda}\left(\left(D_{t}-\lambda_{j}^{(1)}\right) p_{j}^{(1)} v_{\varepsilon}\right) \cdot\left(\overline{p_{j}^{(1)} v_{\varepsilon}}\right)\right\} \\
=\sum_{k=1}^{3} \operatorname{Im}\left\{W_{0}^{2} e^{-A \Lambda}\left(\mathscr{P}_{k} v_{\varepsilon}\right) \cdot\left(\overline{p_{j}^{(1)} v_{\varepsilon}}\right)\right\} \\
\quad+\operatorname{Re}\left\{(-1)^{j} W_{0}^{2} e^{-A \Lambda}\left(\lambda_{2 t}^{(1)}-\lambda_{1 t}^{(1)}\right) v_{\varepsilon} \cdot\left(\overline{p_{j}^{(1)} v_{\varepsilon}}\right)\right\} / 2,
\end{aligned}
$$

$$
\operatorname{Im}\left\{W_{0}^{4} e^{-A \Lambda}\left(D_{t} v_{\varepsilon}\right) \cdot \overline{v_{\varepsilon}}\right\}=\operatorname{Im}\left\{W_{0}^{4} e^{-A \Lambda}\left(p_{1}^{(1)} v_{\varepsilon}\right) \cdot \overline{v_{\varepsilon}}\right\} .
$$

Here we also write $\lambda_{j t}=\partial_{t} \lambda_{j}(t, \xi ; \varepsilon), \lambda_{j t t}=\partial_{t}^{2} \lambda_{j}(t, \xi ; \varepsilon)$ and so forth. (3.1) and (4.1)-(4.5) yield

(4.6) $\partial_{t} \mathscr{E}(t, \xi ; \varepsilon ; A)$

$$
\begin{aligned}
\leq & 3 \Lambda_{t}^{-1} e^{-A \Lambda}|\hat{f}(t, \xi)|^{2} \\
& -\sum_{j=1}^{3}\left[(A-7) \Lambda_{t} e^{-A \Lambda}\left|\mathscr{P}_{j} v_{\varepsilon}\right|^{2}-\Lambda_{t}^{-1} e^{-A \Lambda}\right. \\
& \times\left\{\left|\left(s u b \sigma(P)\left(t, D_{t}, \xi\right)+P_{1}\left(t, D_{t}, \xi\right)+\partial_{t}^{2} \partial_{\tau}^{2} p\left(t, D_{t}, \xi\right) / 6\right) v_{\varepsilon}\right|^{2}\right. \\
+ & \left.\left.\left|P_{0}(t) v_{\varepsilon}\right|^{2}+\sum_{k \neq j}\left|\left(\lambda_{j t}-\lambda_{k t}\right) p_{j, k} v_{\varepsilon}\right|^{2} / 4+\sum_{k \neq j}\left|\left(\lambda_{j t t}-\lambda_{k t t}\right) v_{\varepsilon}\right|^{2} / 36\right\}\right] \\
- & \sum_{j=1}^{3}\left[(A-10) W_{0}^{2} \Lambda_{t} e^{-A \Lambda}\left|p_{j}^{(1)} v_{\varepsilon}\right|^{2}\right\} \\
& -(A-13) W_{0}^{2} \Lambda_{t}^{-1} e^{-A \Lambda}\left\{\sum_{k=1}^{3}\left|\mathscr{P}_{k} v_{\varepsilon}\right|^{2}+\left|\left(\lambda_{2 t}^{(1)}-\lambda_{1 t}^{(1)}\right) v_{\varepsilon}\right|^{2} / 4\right\}\left|v_{\varepsilon}\right|^{2}+W_{0}^{4} \Lambda_{t}^{-1}\left|p_{1}^{(1)} v_{\varepsilon}\right|^{2} .
\end{aligned}
$$


From Lemma 2.6 we can write

$$
\begin{aligned}
& \operatorname{sub} \sigma(P)(t, \tau, \xi)=\sum_{j=1}^{3} b_{1, j}(t, \xi ; \varepsilon) p_{j}(t, \tau, \xi ; \varepsilon), \\
& \min \left\{\min _{s \in \mathscr{R}(\xi /|\xi|)}|t-s|, 1\right\}\left|b_{1, j}(t, \xi ; \varepsilon)\right| \leq C_{T} \quad(1 \leq j \leq 3)
\end{aligned}
$$

for $\xi \in \mathbf{R}^{n} \backslash\{0\}, t \in[0, T] \backslash \mathscr{R}(\xi /|\xi|)$ and $\varepsilon \in \mathbf{R}$, where $C_{T}>0$. Operating $\partial_{\tau}^{2}$ in both sides of (4.7), we have

$$
\partial_{\tau}^{2} P_{2}(t, \tau, \xi)=2 \sum_{j=1}^{3} b_{1, j}(t, \xi ; \varepsilon) .
$$

Since

$$
\begin{aligned}
& \partial_{t} \partial_{\tau} p_{j}(t, \tau, \xi ; \varepsilon)=-\sum_{k \neq j} \lambda_{k t}(t, \xi ; \varepsilon), \\
& \partial_{t} \partial_{\tau}^{2} p(t, \tau, \xi ; \varepsilon)=-2 \sum_{k=1}^{3} \lambda_{k t}(t, \xi ; \varepsilon), \\
& \partial_{t} \partial_{\tau} p_{j}(t, \tau, \xi ; \varepsilon)-\partial_{t} \partial_{\tau}^{2} p(t, \tau, \xi ; \varepsilon) / 3=-\sum_{k \neq j}\left(\lambda_{k t}(t, \xi ; \varepsilon)-\lambda_{j t}(t, \xi ; \varepsilon)\right) / 3,
\end{aligned}
$$

(1.1), (2.18), (4.8) and Lemma 2.6 give

$$
\begin{aligned}
\text { sub } \sigma( & P)(t, \tau, \xi)+P_{1}(t, \tau, \xi)+\partial_{t}^{2} \partial_{\tau}^{2} p(t, \tau, \xi) / 6 \\
= & \sum_{j=1}^{3} b_{1, j}(t, \xi ; \varepsilon) \mathscr{P}_{j}(t, \tau, \xi ; \varepsilon)+P_{1}(t, \tau, \xi)+\partial_{t}^{2} \partial_{\tau}^{2} p(t, \tau, \xi) / 6 \\
& -(i / 6) \sum_{j=1}^{3} \sum_{k \neq j} b_{1, j}(t, \xi ; \varepsilon)\left(\lambda_{k t}(t, \xi ; \varepsilon)-\lambda_{j t}(t, \xi ; \varepsilon)\right) \\
& +(i / 6) \sum_{j=1}^{3} b_{1, j}(t, \xi ; \varepsilon) \partial_{t} \partial_{\tau}^{2} p(t, \tau, \xi) \\
= & \sum_{j=1}^{3} b_{1, j}(t, \xi ; \varepsilon) \mathscr{P}_{j}(t, \tau, \xi ; \varepsilon)+s u b^{2} \sigma(P)(t, \tau, \xi) \\
& -(i / 6) \sum_{j=1}^{3} \sum_{k \neq j} b_{1, j}(t, \xi ; \varepsilon)\left(\lambda_{k t}(t, \xi ; \varepsilon)-\lambda_{j t}(t, \xi ; \varepsilon)\right)
\end{aligned}
$$




$$
\begin{aligned}
= & \sum_{j=1}^{3} b_{1, j}(t, \xi ; \varepsilon) \mathscr{P}_{j}(t, \tau, \xi ; \varepsilon)+\sum_{j=1}^{2} b_{2, j}(t, \xi ; \varepsilon) p_{j}^{(1)}(t, \tau, \xi ; \varepsilon) \\
& -(i / 6) \sum_{j=1}^{3} \sum_{k \neq j} b_{1, j}(t, \xi ; \varepsilon)\left(\lambda_{k t}(t, \xi ; \varepsilon)-\lambda_{j t}(t, \xi ; \varepsilon)\right),
\end{aligned}
$$

where the $b_{2, j}(t, \xi ; \varepsilon)$ satisfy (2.15). If $\{j, k, l\}=\{1,2,3\}$, then we have

$$
\left|\left(\lambda_{j}-\lambda_{k}\right) v_{\varepsilon}\right|^{2}=\left|\left(p_{j, l}-p_{k, l}\right) v_{\varepsilon}\right|^{2} \leq 2\left|p_{j, l} v_{\varepsilon}\right|^{2}+2\left|p_{k, l} v_{\varepsilon}\right|^{2} .
$$

It follows from (2.10) with $m=3, k=2$ and $r=1$ and Lemma 2.5 that there are $b_{j, k, \mu}(t, \xi ; \varepsilon)(\mu=1,2)$ satisfying

$$
\begin{aligned}
& p_{j, k}(t, \tau, \xi ; \varepsilon)=\sum_{\mu=1}^{2} b_{j, k, \mu}(t, \xi ; \varepsilon) p_{\mu}^{(1)}(t, \tau, \xi ; \varepsilon), \\
& \left|b_{j, k, \mu}(t, \xi ; \varepsilon)\right| \leq 1 / \sqrt{2} \quad(\mu=1,2),
\end{aligned}
$$

since

$$
\begin{aligned}
& h_{1}\left(t, \tau, \xi ; p_{\varepsilon}\right)=\sum_{1 \leq j<k \leq 3} p_{j, k}(t, \tau, \xi ; \varepsilon)^{2} \\
& h_{1}\left(t, \tau, \xi ; p_{\varepsilon}^{(1)}\right)=\sum_{\mu=1}^{2} p_{\mu}^{(1)}(t, \tau, \xi ; \varepsilon)^{2} .
\end{aligned}
$$

Therefore, we have

$$
\left|p_{j, k} v_{\varepsilon}\right|^{2} \leq \sum_{\mu=1}^{2}\left|p_{\mu}^{(1)} v_{\varepsilon}\right|^{2} \quad(1 \leq j<k \leq 3) .
$$

It is obvious that

$$
\left|\left(\lambda_{2}^{(1)}-\lambda_{1}^{(1)}\right) v_{\varepsilon}\right|^{2} \leq 2 \sum_{\mu=1}^{2}\left|p_{\mu}^{(1)} v_{\varepsilon}\right|^{2} .
$$

Let us estimate $\Lambda_{t}^{-1} e^{-A \Lambda}\left|\left(s u b \sigma(P)\left(t, D_{t}, \xi\right)+P_{1}\left(t, D_{t}, \xi\right)+\left(\partial_{t}^{2} \partial_{\tau}^{2} p\right)\left(t, D_{t}, \xi\right) / 6\right) v_{\varepsilon}\right|^{2}$. First assume that $\min \left\{\min _{s \in \mathscr{R}(\xi /|\xi|)}|t-s|, 1\right\} \leq\langle\xi\rangle^{-2 / 3}$. Then we have

$$
W_{0}(t, \xi ; \varepsilon) \geq\langle\xi\rangle^{2 / 3} / \sqrt{2} \text {. }
$$

Therefore, we have

$$
\begin{gathered}
\Lambda_{t}^{-1} e^{-A \Lambda}\left|\left(\operatorname{sub} \sigma(P)\left(t, D_{t}, \xi\right)+P_{1}\left(t, D_{t}, \xi\right)+\left(\partial_{t}^{2} \partial_{\tau}^{2} p\right)\left(t, D_{t}, \xi\right) / 6\right) v_{\varepsilon}\right|^{2} \\
\leq C \Lambda_{t} e^{-A \Lambda}\left\{\left|\mathscr{P}_{j} v_{\varepsilon}\right|^{2}+W_{0}^{2}\left|p_{1}^{(1)} v_{\varepsilon}\right|^{2}+W_{0}^{4}\left|v_{\varepsilon}\right|^{2}\right\}
\end{gathered}
$$


for $t \in[0, T], \xi \in \mathbf{R}^{n} \backslash \mathscr{N}$ and $\varepsilon \in E_{0}$, since

$$
\begin{aligned}
& \text { sub } \sigma(P)(t, \tau, \xi)+P_{1}(t, \tau, \xi)+\partial_{t}^{2} \partial_{\tau}^{2} p(t, \tau, \xi) / 6 \\
& \quad=c_{0}(t) \mathscr{P}_{j}(t, \tau, \xi ; \varepsilon)+c_{j, 1}(t, \xi ; \varepsilon) p_{1}^{(1)}(t, \tau, \xi ; \varepsilon)+c_{j, 2}(t, \xi ; \varepsilon), \\
& \left|c_{0}(t)\right| \leq C, \quad\left|c_{j, k}(t, \xi ; \varepsilon)\right| \leq C\langle\xi\rangle^{k} \quad(k=1,2)
\end{aligned}
$$

for $t \in[0, T], \xi \in \mathbf{R}^{n} \backslash \mathscr{N}$ and $\varepsilon \in E_{0}$. Next assume that $\min \left\{\min _{s \in \mathscr{R}(\xi /|\xi|)}|t-s|, 1\right\}$ $\geq\langle\xi\rangle^{-2 / 3}$. Then we have

$$
W_{0}(t, \xi ; \varepsilon) \geq\left(\sqrt{2} \min \left\{\min _{s \in \mathscr{R}(\xi /|\xi|)}|t-s|, 1\right\}\right)^{-1} .
$$

This, together with (4.9)-(4.11), yields

$$
\begin{gathered}
\Lambda_{t}^{-1} e^{-A \Lambda}\left|\left(\operatorname{sub} \sigma(P)\left(t, D_{t}, \xi\right)+P_{1}\left(t, D_{t}, \xi\right)+\left(\partial_{t}^{2} \partial_{\tau}^{2} p\right)\left(t, D_{t}, \xi\right) / 6\right) v_{\varepsilon}\right|^{2} \\
\leq C W_{0}^{2} \Lambda_{t}^{-1} e^{-A \Lambda}\left\{\sum_{j=1}^{3}\left|\mathscr{P}_{j} v_{\varepsilon}\right|^{2}+W_{0}^{2} \sum_{j=1}^{2}\left|p_{j}^{(1)} v_{\varepsilon}\right|^{2}\right. \\
\left.+\sum_{1 \leq j<k \leq 3}\left|\lambda_{j t}-\lambda_{k t}\right|^{2}\left|v_{\varepsilon}\right|^{2}\right\} \\
\leq C^{\prime} \Lambda_{t} e^{-A \Lambda}\left\{\sum_{j=1}^{3}\left|\mathscr{P}_{j} v_{\varepsilon}\right|^{2}+W_{0}^{2} \sum_{j=1}^{2}\left|p_{j}^{(1)} v_{\varepsilon}\right|^{2}+W_{0}^{4}\left|v_{\varepsilon}\right|^{2}\right\}
\end{gathered}
$$

for $t \in[0, T], \xi \in \mathbf{R}^{n} \backslash \mathscr{N}$ and $\varepsilon \in E_{0}$, since

$$
\left|\lambda_{j t}(t, \xi ; \varepsilon)-\lambda_{k t}(t, \xi ; \varepsilon)\right|^{2} \leq W_{0}(t, \xi ; \varepsilon)^{2}\left(\left(\lambda_{j}(t, \xi ; \varepsilon)-\lambda_{k}(t, \xi ; \varepsilon)\right)^{2}+1\right) .
$$

By (4.13) (4.14) is also valid in the case where $\min \left\{\min _{s \in \mathscr{R}(\xi /|\xi|)}|t-s|, 1\right\} \leq$ $\langle\xi\rangle^{-2 / 3}$. We can easily show that

$$
\begin{aligned}
& \Lambda_{t}^{-1} e^{-A \Lambda}\left|P_{0}(t) v_{\varepsilon}\right|^{2} \leq C W_{0}^{4} \Lambda_{t} e^{-A \Lambda}\left|v_{\varepsilon}\right|^{2}, \\
& \Lambda_{t}^{-1} e^{-A \Lambda} \sum_{k \neq j}\left|\left(\lambda_{j t}-\lambda_{k t}\right) p_{j, k} v_{\varepsilon}\right|^{2} \\
& \quad \leq \Lambda_{t}^{-1} e^{-A \Lambda} \sum_{k \neq j}\left(W_{0}^{2}\left|\left(\lambda_{j}-\lambda_{k}\right) p_{j, k} v_{\varepsilon}\right|^{2}+\left|p_{j, k} v_{\varepsilon}\right|^{2}\right) \\
& \quad \leq C^{\prime} \Lambda_{t} e^{-A \Lambda}\left\{\sum_{k=1}^{3}\left|\mathscr{P}_{k} v_{\varepsilon}\right|^{2}+W_{0}^{2} \sum_{k=1}^{2}\left|p_{k}^{(1)} v_{\varepsilon}\right|^{2}\right\} \quad(1 \leq j \leq 3),
\end{aligned}
$$




$$
\begin{aligned}
& \Lambda_{t}^{-1} e^{-A \Lambda} \sum_{k \neq j}\left|\left(\lambda_{j t t}-\lambda_{k t t}\right) v_{\varepsilon}\right|^{2} \\
& \leq \Lambda_{t}^{-1} e^{-A \Lambda} \sum_{k \neq j}\left(W_{0}^{2} W_{1}^{2}\left|\left(\lambda_{j}-\lambda_{k}\right) v_{\varepsilon}\right|^{2}+\left|v_{\varepsilon}\right|^{2}\right) \\
& \leq C W_{0}^{2} \Lambda_{t} e^{-A \Lambda}\left\{\sum_{k=1}^{2}\left|p_{k}^{(1)} v_{\varepsilon}\right|^{2}+W_{0}^{2}\left|v_{\varepsilon}\right|^{2}\right\} \quad(1 \leq j \leq 3), \\
& \text { (4.18) } \quad W_{0}^{2} \Lambda_{t}^{-1} e^{-A \Lambda}\left|\left(\lambda_{2 t}^{(1)}-\lambda_{1 t}^{(1)}\right) v_{\varepsilon}\right|^{2} \\
& \leq W_{0}^{2} W_{1}^{2} \Lambda_{t}^{-1} e^{-A \Lambda}\left(\left|\left(\lambda_{2}^{(1)}-\lambda_{1}^{(1)}\right) v_{\varepsilon}\right|^{2}+\left|v_{\varepsilon}\right|^{2}\right) \\
& \leq 2 W_{0}^{2} \Lambda_{t} e^{-A \Lambda}\left\{\sum_{k=1}^{2}\left|p_{k}^{(1)} v_{\varepsilon}\right|^{2}+W_{0}^{2}\left|v_{\varepsilon}\right|^{2}\right\}
\end{aligned}
$$

for $t \in[0, T], \xi \in \mathbf{R}^{n} \backslash \mathscr{N}$ and $\varepsilon \in E_{0}$. Indeed, (4.15) is obvious. (4.16) follows from (3.12) and (4.11). In (4.17) we use (4.10) and (4.11). (4.18) follows from (4.12). So it follows from (4.6) and (4.14)-(4.18) that there is $A_{0} \geq 1$ satisfying

$$
\partial_{t} \mathscr{E}(t, \tau, \xi ; \varepsilon ; A) \leq 3 \Lambda_{t}^{-1} e^{-A \Lambda}|\hat{f}(t, \xi)|^{2}
$$

for $A \geq A_{0}, t \in[0, T], \xi \in \mathbf{R}^{n} \backslash \mathscr{N}$ and $\varepsilon \in E_{0}$. Therefore, repeating the same arguments as in $\S 3$, we can prove Theorem 1.3.

\section{Some Remarks and Examples}

Let us first consider the validity of the condition (T-L). Let $P(t, \tau, \xi)=$ $(\tau-\lambda(t, \xi))^{3}+b_{2}(t, \tau, \xi)+b_{1}(t, \tau, \xi)+b_{0}(t)$, where

$$
\left\{\begin{array}{l}
\lambda(t, \xi)=\sum_{j=1}^{n} \lambda_{j}(t) \xi_{j} \\
b_{2}(t, \tau, \xi)=b_{2,0}(t) \tau^{2}+\sum_{j=1}^{n} b_{2, j}(t) \tau \xi_{j}+\sum_{|\alpha|=2} b_{2, \alpha}(t) \xi^{\alpha} \\
b_{1}(t, \tau, \xi)=b_{1,0}(t) \tau+\sum_{j=1}^{n} b_{1, j}(t) \xi_{j} .
\end{array}\right.
$$

We assume that the $\lambda_{j}(t)$ are real-valued and that the $\lambda_{j}(t)$, the $b_{j, k}(t)$ and $b_{0}(t)$ belong to $C^{\infty}([0, \infty))$. It is well-known that the Cauchy problem for $P\left(t, D_{t}, D_{x}\right)$ is $C^{\infty}$ well-posed if and only if $P\left(t, D_{t}, D_{x}\right)$ can be represented as follows:

$$
P\left(t, D_{t}, D_{x}\right)=\left(D_{t}-\lambda\left(t, D_{x}\right)\right)^{3}+\sum_{j=0}^{2} c_{j}(t)\left(D_{t}-\lambda\left(t, D_{x}\right)\right)^{j},
$$

where $c_{j}(t) \in C^{\infty}([0, \infty))(0 \leq j \leq 2)$ (see, e.g., [4] and [18]). 
The following theorem insists that the condition $(\mathrm{T}-\mathrm{L})$ is a reasonable and likely condition for $C^{\infty}$ well-posedness.

THEOREM 5.1. The Cauchy problem for $P\left(t, D_{t}, D_{x}\right)$ is $C^{\infty}$ well-posed if and only if the condition (T-L) with $\mathscr{R}(\xi)=\varnothing$ is satisfied.

ProOF. It is obvious that

$$
\begin{aligned}
& (\tau-\lambda(t, \xi)) \circ(\tau-\lambda(t, \xi))=(\tau-\lambda(t, \xi))^{2}+i \partial_{t} \lambda(t, \xi) \\
& (\tau-\lambda(t, \xi)) \circ(\tau-\lambda(t, \xi)) \circ(\tau-\lambda(t, \xi)) \\
& \quad=(\tau-\lambda(t, \xi))^{3}+3 i \partial_{t} \lambda(t, \xi) \cdot(\tau-\lambda(t, \xi))+\partial_{t}^{2} \lambda(t, \xi) .
\end{aligned}
$$

Therefore, we have

$$
\begin{aligned}
P\left(t, D_{t}, D_{x}\right)= & \left(D_{t}-\lambda\left(t, D_{x}\right)\right)^{3}+b_{2,0}(t)\left(D_{t}-\lambda\left(t, D_{x}\right)\right)^{2} \\
& +b_{1,0}(t)\left(D_{t}-\lambda\left(t, D_{x}\right)\right)+\tilde{b}_{2}\left(t, D_{t}, D_{x}\right)+\tilde{b}_{1}\left(t, D_{t}, D_{x}\right)+b_{0}(t),
\end{aligned}
$$

where

$$
\begin{aligned}
& \tilde{b}_{2}(t, \tau, \xi)=b_{2}(t, \tau, \xi)-b_{2,0}(t)(\tau-\lambda(t, \xi))^{2}-3 i \partial_{t} \lambda(t, \xi) \cdot(\tau-\lambda(t, \xi)) \\
& \tilde{b}_{1}(t, \tau, \xi)=b_{1}(t, \tau, \xi)-b_{1,0}(t)(\tau-\lambda(t, \xi))-i b_{2,0}(t) \partial_{t} \lambda(t, \xi)-\partial_{t}^{2} \lambda(t, \xi) .
\end{aligned}
$$

On the other hand, we have

$$
\begin{aligned}
& h_{2}(t, \tau, \xi)^{1 / 2}=\sqrt{3}(\tau-\lambda(t, \xi))^{2}, \quad h_{1}(t, \tau, \xi)^{1 / 2}=\sqrt{3}|\tau-\lambda(t, \xi)|, \\
& \operatorname{sub\sigma } \sigma(P)(t, \tau, \xi)=b_{2}(t, \tau, \xi)-3 i \partial_{t} \lambda(t, \xi) \cdot(\tau-\lambda(t, \xi)), \\
& \operatorname{sub}^{2} \sigma(P)(t, \tau, \xi)=b_{1}(t, \tau, \xi)-\partial_{t}^{2} \lambda(t, \xi)-i b_{2,0}(t) \partial_{t} \lambda(t, \xi) .
\end{aligned}
$$

This, together with (5.2) and (5.3), shows that

$$
\tilde{b}_{2}(t, \tau, \xi) \equiv \tilde{b}_{1}(t, \tau, \xi) \equiv 0
$$

if and only if the condition (T-L) is satisfied, since (T-L) implies that $\operatorname{sub} \sigma(P)(t, \tau, \xi)$ and $\operatorname{sub}^{2} \sigma(P)(t, \tau, \xi)$ are divided by $(\tau-\lambda(t, \xi))^{2}$ and $(\tau-\lambda(t, \xi))$, respectively. 
Finally we shall give two simple examples.

Example 5.2. Let $P(t, \tau, \xi)=\left(\tau^{2}-a(t)|\xi|^{2}\right) \tau+b_{2}(t, \tau, \xi)+b_{1}(t, \tau, \xi)+b_{0}(t)$, where $b_{j}(t, \tau, \xi)(j=1,2)$ and $b_{0}(t)$ are as in (5.1) and $a(t)$ is real analytic in a neighborhood of $[0, \infty)$. Assume that $a(t) \geq 0$ for $t \in[0, \infty)$. Then the conditions (A-1), (A-2) and (T) are satisfied. A simple calculation yields

$$
\begin{aligned}
& h_{2}(t, \tau, \xi)=3 \tau^{4}+a(t)^{2}|\xi|^{4}, \\
& h_{1}(t, \tau, \xi)=3 \tau^{2}+2 a(t)|\xi|^{2}, \\
& \operatorname{sub} \sigma(P)(t, \tau, \xi)=b_{2}(t, \tau, \xi)-(i / 2) \partial_{t} a(t) \cdot|\xi|^{2}, \\
& \operatorname{sub}^{2} \sigma(P)(t, \tau, \xi)=b_{1}(t, \tau, \xi) .
\end{aligned}
$$

Therefore, we have

$$
\begin{aligned}
\left(\tau^{2}+\sqrt{a(t)}|\tau||\xi|+a(t)|\xi|^{2}\right) / 5 & \leq h_{2}(t, \tau, \xi)^{1 / 2} \\
& \leq 2\left(\tau^{2}+\sqrt{a(t)}|\tau||\xi|+a(t)|\xi|^{2}\right), \\
|\tau|+\sqrt{a(t)}|\xi| \leq h_{1}(t, \tau, \xi)^{1 / 2} \leq & 2(|\tau|+\sqrt{a(t)}|\xi|) .
\end{aligned}
$$

Let $t_{k} \in[0, \infty)$ be a zero of $a(t)$ of order $v_{k}(k=1,2,3, \ldots)$, where $0 \leq t_{1}<t_{2}<$ $t_{3}<\cdots$. Taking $\mathscr{R}(\xi)=\left\{t_{1}, t_{2}, t_{3}, \ldots\right\}$ we can see that the condition (T-L) is satisfied if and only if

$$
\begin{aligned}
& b_{2, j}(t)=O\left(\left(t-t_{k}\right)^{v_{k} / 2-1}\right) \quad \text { as } t \rightarrow t_{k} \quad(1 \leq j \leq n), \\
& b_{2, \alpha}(t)=O\left(\left(t-t_{k}\right)^{v_{k}-1}\right) \quad \text { as } t \rightarrow t_{k} \quad(|\alpha|=2), \\
& b_{1, j}(t)=O\left(\left(t-t_{k}\right)^{v_{k} / 2-2}\right) \quad \text { as } t \rightarrow t_{k} \quad(1 \leq j \leq n)
\end{aligned}
$$

for $k=1,2,3, \ldots$

Let $S$ be a subset of $[0, \infty) \times S^{n-1}$, and define the condition $(\mathrm{T}-\mathrm{L})_{S}$ by replacing $[0, T] \times \mathbf{R} \times S^{n-1}$ with $\left\{(t, \tau, \xi) \in[0, T] \times \mathbf{R} \times S^{n-1} ;(t, \xi) \in S\right\}$ in the condition (T-L).

ExAmPle 5.3. Let $P(t, \tau, \xi)=(\tau-\lambda(t, \xi)) \tau^{2}+b_{2}(t, \tau, \xi)+b_{1}(t, \tau, \xi)+b_{0}(t)$, where $\lambda(t, \xi)=t^{\kappa_{1}} \xi_{1}+t^{\kappa_{2}} \xi_{2}, \kappa_{1}, \kappa_{2} \in \mathbf{Z}_{+}, \kappa_{1} \leq \kappa_{2}$ and $b_{j}(t, \tau, \xi) \quad(j=1,2)$ and $b_{0}(t)$ are as in (5.1). A simple calculation yields 


$$
\begin{aligned}
& h_{2}(t, \tau, \xi)=\tau^{2}\left(\tau^{2}+2(\tau-\lambda(t, \xi))^{2}\right), \\
& h_{1}(t, \tau, \xi)=2 \tau^{2}+(\tau-\lambda(t, \xi))^{2}, \\
& \operatorname{sub} \sigma(P)(t, \tau, \xi)=b_{2}(t, \tau, \xi)-i \partial_{t} \lambda(t, \xi) \cdot \tau, \\
& \operatorname{sub}^{2} \sigma(P)(t, \tau, \xi)=b_{1}(t, \tau, \xi)-\partial_{t}^{2} \lambda(t, \xi) / 3-(i / 3) b_{2,0}(t) \partial_{t} \lambda(t, \xi) .
\end{aligned}
$$

Therefore, we have

$$
\begin{aligned}
& \left(\tau^{2}+|\lambda(t, \xi)||\tau|\right) / 3 \leq h_{2}(t, \tau, \xi)^{1 / 2} \leq 3\left(\tau^{2}+|\lambda(t, \xi)||\tau|\right) \\
& (|\tau|+|\lambda(t, \xi)|) / 2 \leq h_{1}(t, \tau, \xi)^{1 / 2} \leq 2(|\tau|+|\lambda(t, \xi)|) .
\end{aligned}
$$

It is obvious that

(5.4) $\quad b_{2, j}(t) \equiv b_{1, j}(t) \equiv 0 \quad(3 \leq j \leq n) \quad$ and $\quad b_{2, \alpha}(t) \equiv 0 \quad(|\alpha|=2)$

if the condition (T-L) is satisfied. Let us first consider the case where $\kappa_{1}=\kappa_{2}$. Then it is easy to see that the condition (T-L) with $\mathscr{R}(\xi)=\{0\}$ is satisfied if and only if

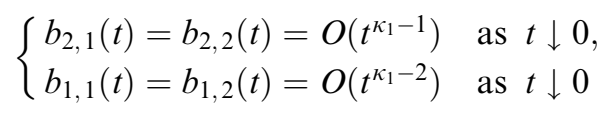

and (5.4) is satisfied. Next consider the case where $\kappa_{1}<\kappa_{2}$. Put

$$
\begin{aligned}
& S_{1}=\left\{(t, \xi) \in[0, \infty) \times S^{n-1} ; \xi_{1} \xi_{2} \geq 0\right\}, \\
& S_{2}=\left\{(t, \xi) \in[0, \infty) \times S^{n-1} ; \xi_{1} \xi_{2}<0 \text { and } 0 \leq t^{\kappa} \leq\left|\xi_{1} / \xi_{2}\right| / 2\right\}, \\
& S_{3}=\left\{(t, \xi) \in[0, \infty) \times S^{n-1} ; \xi_{1} \xi_{2}<0 \text { and } t^{\kappa} \geq\left|\xi_{1} / \xi_{2}\right| / 2\right\}
\end{aligned}
$$

where $\kappa=\kappa_{2}-\kappa_{1}$. If $(t, \xi) \in S_{1}$, then we have

$$
|\lambda(t, \xi)|=t^{\kappa_{1}}\left|\xi_{1}\right|+t^{\kappa_{2}}\left|\xi_{2}\right| .
$$

Therefore, the condition $(\mathrm{T}-\mathrm{L})_{S_{1}}$ with $\mathscr{R}(\xi)=\{0\}$ is satisfied if and only if

$$
b_{2, j}(t)=O\left(t^{\kappa_{j}-1}\right) \quad \text { and } \quad b_{1, j}(t)=O\left(t^{\kappa_{j}-2}\right) \quad \text { as } t \downarrow 0
$$

for $j=1,2$ and (5.4) is satisfied. If $(t, \xi) \in S_{2}$ and $\mathscr{R}(\xi)=\{0\}$, then we have

$$
\begin{aligned}
\left(t^{\kappa_{1}}\left|\xi_{1}\right|+t^{\kappa_{2}}\left|\xi_{2}\right|\right) / 4 & \leq|\lambda(t, \xi)|=t^{\kappa_{1}}\left|\xi_{2}\right|\left(\left|\xi_{1} / \xi_{2}\right|-t^{\kappa}\right) \\
& \leq t^{\kappa_{1}}\left|\xi_{1}\right|+t^{\kappa_{2}}\left|\xi_{2}\right| .
\end{aligned}
$$


This implies that $(\mathrm{T}-\mathrm{L})_{S_{2}}$ with $\mathscr{R}(\xi)=\{0\}$ is satisfied if and only if (5.4) and (5.5) are satisfied, since

$$
\begin{aligned}
& \left|\partial_{t} \lambda(t, \xi)\right| \leq \kappa_{2}\left(t^{\kappa_{1}-1}\left|\xi_{1}\right|+t^{\kappa_{2}-1}\left|\xi_{2}\right|\right), \\
& \left|\partial_{t}^{2} \lambda(t, \xi)\right| \leq \kappa_{2}\left(\kappa_{2}-1\right)\left(t^{\kappa_{1}-2}\left|\xi_{1}\right|+t^{\kappa_{2}-2}\left|\xi_{2}\right|\right) .
\end{aligned}
$$

If $(t, \xi) \in S_{3}$ and $\mathscr{R}(\xi)=\left\{\left|\xi_{1} / \xi_{2}\right|^{1 / \kappa}\right\}$, then we have

$$
\begin{aligned}
& \min \left\{\min _{s \in \mathscr{R}(\xi)}|t-s|, 1\right\}=\min \left\{|t-| \xi_{1} /\left.\xi_{2}\right|^{1 / \kappa} \mid, 1\right\} \\
& \min \left\{\min _{s \in \mathscr{R}(\xi)}|t-s|^{2}, 1\right\}=\min \left\{|t-| \xi_{1} /\left.\left.\xi_{2}\right|^{1 / \kappa}\right|^{2}, 1\right\} \\
& |t-| \xi_{1} /\left.\xi_{2}\right|^{1 / \kappa} \mid\left(t^{\kappa_{1}-1}\left|\xi_{1}\right|+t^{\kappa_{2}-1}\left|\xi_{2}\right|\right) / 3 \\
& \quad \leq|\lambda(t, \xi)|=t^{\kappa_{1}}\left|\xi_{2}\right||t-| \xi_{1} /\left.\xi_{2}\right|^{1 / \kappa} \mid\left(t^{\kappa-1}+\left|\xi_{1} / \xi_{2}\right|^{1 / \kappa} t^{\kappa-2}+\cdots+\left|\xi_{1} / \xi_{2}\right|^{1-1 / \kappa}\right) \\
& \quad \leq\left(2^{1 / \kappa}-1\right)^{-1} t^{\kappa_{2}-1}\left|\xi_{2}\right||t-| \xi_{1} /\left.\xi_{2}\right|^{1 / \kappa} \mid \\
& \quad \leq\left(2^{1 / \kappa}-1\right)^{-1}|t-| \xi_{1} /\left.\xi_{2}\right|^{1 / \kappa} \mid\left(t^{\kappa_{1}-1}\left|\xi_{1}\right|+t^{\kappa_{2}-1}\left|\xi_{2}\right|\right) .
\end{aligned}
$$

Therefore, the condition (T-L) $S_{3}$ with $\mathscr{R}(\xi)=\left\{\left|\xi_{1} / \xi_{2}\right|^{1 / \kappa}\right\}$ is satisfied if and only if (5.4) and (5.5) are satisfied, since

$$
\begin{aligned}
& \left|\partial_{t} \lambda(t, \xi)\right| \leq \kappa_{2}\left(t^{\kappa_{1}-1}\left|\xi_{1}\right|+t^{\kappa_{2}-1}\left|\xi_{2}\right|\right), \\
& |t-| \xi_{1} /\left.\xi_{2}\right|^{1 / \kappa}|| \partial_{t}^{2} \lambda(t, \xi)|\leq t| \partial_{t}^{2} \lambda(t, \xi) \mid \leq \kappa_{2}\left(\kappa_{2}-1\right)\left(t^{\kappa_{1}-1}\left|\xi_{1}\right|+t^{\kappa_{2}-1}\left|\xi_{2}\right|\right) .
\end{aligned}
$$

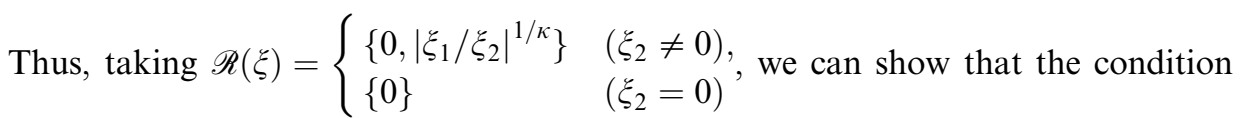
(T-L) is satisfied if and only if (5.4) and (5.5) are satisfied. Moreover, it is easy to see that (5.4) and (5.5) are satisfied if the condition (T-L) is satisfied for some $\mathscr{R}(\xi)$.

\section{References}

[ 1 ] Colombini, F. and Orrú, N., Well-posedness in $C^{\infty}$ for some weakly hyperbolic equations, J. Math. Kyoto Univ. 39 (1999), 399-420.

[2] Colombini, F. and Taglialatela, G., Well-posedness for hyperbolic higher order operators with finite degeneracy, J. Math. Kyoto Univ. 46 (2006), 833-877.

[ 3 ] D'Ancona, P. and Kinoshita, T., On the wellposedness of the Cauchy problem for weakly hyperbolic equations of higher order, Math. Nachr. 278 (2005), 1147-1162. 
[4] Flaschka, H. and Strang, G., The correctness of the Cauchy problem, Advances in Math. 6 (1971), 347-379.

[ 5 ] Hitotumatu, S., Theory of Analytic Functions of Several Complex Variables, Baifu-Kan, 1960. (Japanese)

[6] Hörmander, L., The Analysis of Linear Partial Differential Operators II, Springer, BerlinHeidelberg-New York-Tokyo, 1983.

[7] Ishida, H., Levi conditions to the Gevrey well-posedness for hyperbolic operators of higher order, J. Math. Kyoto Univ. 49 (2009), 173-191.

[ 8 ] Jackson, R. V. J., Canonical Differential Operators and Lower-order Symbols, Memoirs of AMS, No. 135, 1973.

[9] Kajitani, K. and Wakabayashi, S., Microlocal a priori estimates and the Cauchy problem I, Japanese J. Math. 19 (1993), 353-418.

[10] Nuij, W., A note on hyperbolic polynomials, Math. Scand. 23 (1968), 69-72.

[11] Svensson, S. L., Necessary and sufficient conditions for the hyperbolicity of polynomials with hyperbolic principal part, Ark. Mat. 8 (1969), 145-162.

[12] Wakabayashi, S., The Cauchy problem for operators with constant coefficient hyperbolic principal part and propagation of singularities, Japanese J. Math. 6 (1980), 179-228.

[13] Wakabayashi, S., Singularities of solutions of the Cauchy problem for hyperbolic systems in Gevrey classes, Japanese J. Math. 11 (1985), 157-201.

[14] Wakabayashi, S., Remarks on hyperbolic polynomials, Tsukuba J. Math. 10 (1986), 17-28.

[15] Wakabayashi, S., Generalized flows and their applications, Proc. NATO ASI on Advances in Microlocal Analysis, Series C, D. Reidel, 1986, pp363-384.

[16] Wakabayashi, S., On the Cauchy problem for hyperbolic operators of second order whose coefficients depend only on the time variable, J. Math. Soc. Japan 62 (2010), 95-133.

[17] Wakabayashi, S., On the Cauchy problem for second-order hyperbolic operators with the coefficients of their principal parts depending only on the time variable, Funkcialaj Ekvacioj 55 (2012), 99-136.

[18] Zeman, M., The well-posedness of the Cauchy problem for partial differential equations with multiple characteristics, Comm. Partial Differential Equations 2 (1977), 223-249.

\author{
Seiichiro Wakabayashi \\ (professor emeritus) \\ Institute of Mathematics \\ University of Tsukuba \\ Tsukuba, Ibaraki 305-8571 \\ Japan \\ e-mail: wkbysh@math.tsukuba.ac.jp
}

